\title{
Estudios de impacto económico en infraestructuras del transporte: el caso portuario
}

\author{
DE LA PEÑA ZARZUELO, IGNACIO \\ Escuela de Ingenieros de Caminos, Canales y Puertos. Universidad Politécnica de Madrid \\ Correo electrónico: i.delapeña@upm.es \\ FREIRE SEOANE, MARÍa JEsús \\ Facultad de Economía y Empresa. Universidade A Coruña \\ Correo electrónico: maje@udc.es \\ LÓPEZ BERMÚDEZ, BEATRIZ \\ Facultad de Economía y Empresa. Universidade A Coruña \\ Correo electrónico: beatriz.lopez2@udc.es
}

\section{RESUMEN}

Los Estudios de Impacto Económico en puertos son una herramienta muy extendida que empezaron a elaborarse en la década de los años 60 en Estados Unidos. Desde entonces, estos estudios han ido depurándose, eliminando algunas de sus limitaciones y restricciones y convergiendo hacia una metodología estándar que permiten realizar comparaciones entre los efectos de distintos puertos. El artículo analiza la evolución histórica de dichos estudios, indicando las diferentes variantes y sus limitaciones metodológicas y concluye con un resumen de los resultados obtenidos tanto a nivel internacional como en el caso particular del sistema portuario español. Se concluye el papel que tanto el tamaño del puerto como su estructura de tráficos tienen en los impactos que éste genera en términos de empleo, valor añadido y productividad.

Palabras clave: evaluación de impacto económico, matriz de insumo-producto, Leontief, economía portuaria, productividad portuaria.

Clasificación JEL: C67; R15; R42; L90; A10.

MSC2010: 90B06; 93B15; 93D25; 91B50; 91B64; 62P30.

Artículo recibido el 19 de julio de 2019 y aceptado el 1 de noviembre de 2019 


\title{
Economic impact studies on transportation infrastructures: the port case
}

\begin{abstract}
Economic Impact Studies in ports are a very widespread tool that began to be developed in the decade of the 60s in the United States. Since then, these studies have been refined, eliminating some of their limitations and restrictions and converging towards a standard methodology that allows comparisons between the effects of different ports. The article analyzes the historical evolution of these studies, indicating the different variants and their methodological limitations and concludes with a summary of the results obtained both internationally and in the particular case of the Spanish port system. It concludes the role that both the size of the port and its traffic structure have in the impacts it generates in terms of employment, added value and productivity.
\end{abstract}

Keywords: economic impact assement, input - output matrix, Leontief, port economics, port productivity.

JEL classification: C67; R15; R42; L90; A10.

MSC2010: 90B06; 93B15; 93D25; 91B50; 91B64; 62P30.

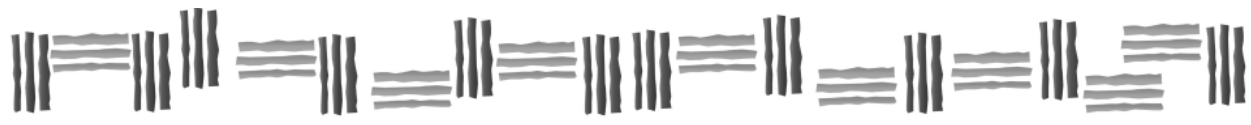




\section{Introducción.}

Los Estudios de Impacto Económico (EIE) están ampliamente extendidos en muchos sectores. En estos estudios se trata de analizar la repercusión económica que una determinada actividad tiene a cierta escala geográfica (local, comarcal, regional, nacional, supranacional...) basándose en la medida de los efectos de dicha actividad en determinados indicadores económicos, tales como el Valor Añadido Bruto (VAB), participación en el PIB, el empleo generado o la recaudación fiscal generada.

No son pocos los detractores que con carácter general los acusan de corresponder a estudios de parte, contratados por grupos de interés con el objetivo de poner en valor los beneficios de la actividad que desarrollan sin seguir procedimientos rigurosos y con escasa base económica, donde se sobrevaloran los efectos positivos y se ocultan las externalidades o dejan sin considerar otros elementos como los costes de oportunidad.

La aplicación de EIE en el campo de transportes como complemento en las fases de evaluación de proyectos de inversión o en el marco de procedimientos de evaluación ambiental es relativamente común. Dentro de esta área de actividad, el desarrollo específico de Estudio de Impacto Económico de Puertos (EIEP) cuenta con una larga tradición que comenzó a mediados de la década de los 60 en puertos de Estados Unidos y que a lo largo de más de 50 años se ha extendido a todo el mundo usando diferentes aproximaciones metodológicas que han ido convergiendo y estandarizándose lo que permite formular estudios comparativos entre distintos puertos o entre las diferentes actividades que en un puerto se desarrollan.

En el presente artículo se da repaso a estas técnicas, primero realizando una revisión bibliográfica y analizando su evolución histórica y clasificación (sección 2), para después discutir su utilización y justificación realizando una revisión crítica de los mismos (sección 3), comparar los extendidos EIE mediante metodología Input-Output (I-O) con los estudios Coste - Beneficio (sección 4) y finalizar con una revisión de los resultados obtenidos en diversos estudios, conclusiones y recomendaciones para próximas líneas de investigación (sección 5).

\section{Revisión bibliográfica. El desarrollo de Estudios de Impacto Económico en puertos: evolución historia y clasificación.}

\subsection{Los orígenes: primeros estudios de la década de los $\mathbf{7 0}$.}

La importancia económica de los puertos y su rol en el desarrollo de una región o nación resulta un hecho incontestable que ha sido objeto de abundante debate científico (Dwarakisha \& Salima, 2015). Por ello, los estudios que tratan de medir de una manera objetiva el impacto económico de los puertos en la zona de influencia en la que se asientan resultan numerosos tanto desde el punto de vista teórico como práctico y desde diferentes enfoques metodológicos o distintos alcances territoriales.

El desarrollo de los EIEP, o al menos lo que respecta a su difusión científica, comenzó en Estados Unidos a mediados de la década de los 60 (Kaufmann, 1979). Pioneros fueron los estudios de Rudy (1961) en el puerto de Seattle, Anderson (1964) en el puerto de Ensenada (California, USA), Roesti et al. (1964) en el distrito portuario de Sacramento-Yolo (California, USA) y Schenker (1965) con su trabajo sobre el impacto de un puerto en su "comunidad urbana". Otras experiencias prácticas le siguen, como las de Conn et al. (1966) en el puerto de Missisipi, Schenker (1967) sobre el puerto de Milwaukee (Milwaukee, USA) o las de Hille y Suelflow (1969) sobre el puerto de Baltimore (Maryland, USA).

Como excepción a todos estos estudios en Estados Unidos, Erbuth (1966) realiza un primer EIEP en Europa analizando los puertos de Hamm y Neuss (Alemania). 
Los estudios se multiplicaron en la primera parte de la década de los 70 empezando con Schenker et al. (1970) quienes asientan las bases para la realización de futuros estudios cuantitativos del impacto de los puertos en su hinterland, Watson (1970) quienes estudian el caso del puerto de Portland (Oregon, USA); Francis (1970) quien aborda el estudio del impacto del puerto de Vancouber (Brithish Columbia, Canadá); Rose (1970) para quien el escenario experimental fue el puerto de Galveston (Texas, USA), las investigaciones de la Port of Seattle Commision (1971) centrados en el puerto Seattle (Seattle, USA); del propio Schenker (1972) quien analizó Green Bay (Wisconsin, USA), de Condor y Tynerson (1972) sobre los puertos de Oregón (Oregon, USA) y de Bragg y Bradley (1972) quienes enlazan su EIEP con un análisis coste-beneficio pero que cuenta con la limitación de circunscribirse a las actividades de una refinería en Texas.

Los estudios continúan con Gruen et al. (1972) en San Francisco (California, USA); Dunphy y Chang (1973) quienes estudian el puerto Mobile (Alabama, USA); Ryan y Adams (1973) en Corpus Christi (Texas, USA); Bragg (1974) que se ocupa del puerto de Gavelston (Texas, USA), Pender y Wilder (1974) quienes se centran en los puertos de Carolina del Sur (Carolina del Sur, USA), Whitaker-Mohn (1974) sobre el puerto de Sacramento.

Stevenson \& Kellog Ltd (1975) estudian el puerto de Vancouver y presentan una interesante separación del impacto categorizado por tipo de mercancía, por tipo de buque y por tipo de instalación portuaria que será seguida en el futuro y hoy en día es una clara tendencia en los EIEP.

Finalmente, Carew (1975) para quienes el escenario experimental es el puerto de Stockton (California, USA), centran su análisis haciendo énfasis en la naturaleza estática de estos estudios (valoran los impactos en relación a los años 1973 y 1974).

Como denominador común, todos los estudios arriba referenciados trataban de evaluar el impacto total (o repercusión media por tonelada) que las actividades portuarias producían en el empleo, salarios, recaudación fiscal, compras desde la industria portuaria al resto de sectores, ingresos de capital, inversiones o en la renta de una región.

Para ello utilizan básicamente tres técnicas que implementan de manera independiente o complementaria: i) análisis de base económica, ii) análisis de ingresos y gastos y, iii) utilización de indicadores y multiplicadores provenientes de estudios de otros puertos.

Desde el punto de su alcance, la mayoría de estos primeros estudios trataban de evaluar el impacto económico en relación a terminales o puertos existentes. Solamente, Bragg y Bradley (1972) trataban de evaluar el futuro impacto de un puerto de aguas profundas de nuevo construcción en Texas (USA), campo que años más tarde tendría un largo recorrido práctico y académico con el objetivo de valorar la bondad de determinadas políticas de inversión.

Esta última variante de EIEP que tratan de evaluar el impacto de determinada inversión (más que de las actividades que en un puerto se desarrollan y los tráficos que en él se mueven) se puede abordar desde dos aproximaciones. En algunos estudios se estima el impacto en términos brutos considerando que la economía futura es idéntica a la presente y midiendo los resultados entre la situación presente y la futura en dos escenarios distintos: con y sin la infraestructura portuaria. En otros se calcula el impacto neto estableciendo hipótesis en relación a cómo va a evolucionar la economía, proyectando esta situación al año horizonte de análisis y midiendo como afecta la nueva infraestructura portuaria a la futura situación económica. Kaufmann (1979) defiende que este último es el enfoque más acertado debido a la posibilidad de que las existencias de unas $\mathrm{u}$ otras infraestructuras portuarias alteren significativamente la economía regional (reduciendo o aumentando los costes de transporte elemento que debe ser considerado en los EIEP).

Las críticas a estos primeros EIEP llegan de la mano de Waters (1977), para quien estos primeros estudios no podían ser utilizados como una herramienta de planificación portuaria y 
adolecían de defectos teóricos al no cuantificar los efectos marginales o incrementales derivadas de las inversiones públicas en el puerto; no capturar los cambios que en las relaciones entre agentes pueden venir derivados de cambios tecnológicos; ignorar que el puerto es un elemento de un sistema productivo no teniendo en consideración el impacto de las importaciones en la región portuaria; o incorporar hipótesis cuestionables como la que se refiere a que las exportaciones de una región incrementan los ingresos de la misma permaneciendo los precios constantes o que los gastos de la industria portuaria tienen un efecto inducido en los ingresos de dicha región.

Este autor para resolver parte de estas carencias propone que sean utilizadas las matrices del modelo económico regional utilizando la metodología de tablas input-output (TIO) en el contexto de desarrollo de EIEP y destaca las ventajas de este análisis frente a otros implementados hasta la fecha.

Un año más tarde, Chang (1978) sale en defensa de los EIEP y pone de manifiesto como mucho de los defectos metodológicos que señala Waters (1977), siendo ciertos, se deben a la propia naturaleza estática de los modelos aplicados y de una lectura no adecuada de los mismos por parte de éste. Efectivamente, estos modelos deben entenderse como ejercicios prácticos para analizar el impacto de las actividades portuarias en un año en particular y no tienen como objeto ser implementados como una herramienta de planificación portuaria sino como un instrumento para poner el valor las actividades del puerto y el impacto positivo de las mismas sobre la región en que se asientan. En el modelo de este autor, que ilustra con un ejemplo práctico aplicado nuevamente al puerto de Mobile (Alabama, USA), se defiende su utilización para justificar las necesarias inversiones a realizar en un puerto y propone que sean utilizados para testar la rentabilidad de las inversiones a acometer. Para ello deben ser utilizadas en combinación con análisis de productividad y niveles de utilización del puerto (capacidad real y deseada tras la ampliación) de tal manera que los EIEP no sustituyan sino complementen otros estudios de costebeneficio.

De igual opinión es Kaufmann (1979) para quien los EIEP deben contribuir a dar información complementaria en los análisis coste-beneficio dentro del proceso de decisión implícito a nuevas políticas, programas o actuaciones de inversión a escala regional. Este autor hace un meritorio recopilación de 20 EIEP publicados a la fecha con el objetivo de analizar la bondad de los diferentes métodos utilizados. El autor utiliza dos criterios: i) valor real de la información proporcionada por dichos estudios y ii) costes comparativos de diferentes metodologías. Así mismo profundiza en cuatro elementos básicos de los estudios: i) cómo determinan los ahorros del coste de transporte a escala local y regional, ii) cómo evalúan los impactos primarios, iii) cómo abordan los impactos secundarios $\mathrm{y}$, finalmente, iv) cómo determinan los efectos multiplicadores. Argumenta que los EIEP son una herramienta eficaz que puede ser utilizada para fijar criterios de política portuaria, pero incide en que estos estudios deben ser leídos correctamente comprendiendo lo que cubren, lo que omiten y cuál es su propósito (toda vez que los mismos se realizan para ser analizados por un conjunto amplio de grupos de interés con diferentes objetivos y perspectivas).

Como resumen de este primer periodo que abarca las décadas de los 60 y 70 se puede observar que existen diferencias sustanciales que impedían comparar los resultados de diferentes EIEP. Entre las diferencias más significativas:

a) No todos los estudios determinan los impactos fijándose en los mismos parámetros incluyendo diferentes indicadores (Tabla 1).

b) No todos los autores identifican claramente en su estudio cuáles son las empresas que producen los impactos primarios del puerto y cuando lo hacen existen diferencias muy significativas (Tabla 2).

c) El alance de los trabajos no es el mismo. Mientras todos los estudios tratan de evaluar impactos directos, no todos calculan los efectos indirectos y muy pocos los efectos en 
términos del ahorro de coste de transporte por el efecto de la presencia del puerto (Tabla $3)$.

d) Existen diferencias metodológicas sustanciales a la hora de determinar los efectos secundarios (Tabla 4).

e) También existen diferencias de escala geográfica para la cuantificación de impactos que se miden en términos local, comarcal, regional o nacional.

f) Falta de homogeneidad en la fuente de datos utilizados en los estudios que van desde trabajo de campo y encuestas directas a las empresas, consulta de otros estudios económicos y asunción de ciertas hipótesis tomadas de éstos, análisis de cuentas y registros públicos de empresas, tablas TIO, etc.

Tabla 1. Indicadores de Impactos (primarios y secundarios) utilizados en EIEP.

\begin{tabular}{|c|c|c|c|c|c|c|c|c|c|c|c|}
\hline \multirow[t]{2}{*}{ Autor } & \multicolumn{11}{|c|}{ INDICADOR } \\
\hline & 1 & 2 & 3 & 4 & 5 & 6 & 7 & 8 & 9 & 10 & 11 \\
\hline The Port of Portland (n/a) & $\bullet$ & & $\bullet$ & & & & & & & & $\bullet$ \\
\hline Rudy (1961) & $\bullet$ & & • & - & & & & & $\bullet$ & & \\
\hline Roesti et al. (1964) & $\bullet$ & & & & & & & & & & \\
\hline Conn (1966) & & - & & & & & & & $\bullet$ & & \\
\hline Erbuth (1966) & & & & & & & & - & & & \\
\hline Hille y Suelflow (1970) & & & & - & & & & - & & & \\
\hline Bragg (1972) & $\bullet$ & & & - & & & & & & & \\
\hline Gruen, Gruen \& Associates (1972) & $\bullet$ & $\bullet$ & & & & & & & & & \\
\hline Schenker. (1972) & & & & - & & & & & & & \\
\hline Oregon Department of Transportation (1973) & - & & • & & & & & & & & \\
\hline Ryan y Adams (1973) & $\bullet$ & - & & & & & & & & & \\
\hline Dunphy (1974) & • & - & & & & & & & $\bullet$ & & \\
\hline Whitaker-Mohn (1974) & $\bullet$ & $\bullet$ & & & $\bullet$ & & & & & & \\
\hline Carew (1975) & $\bullet$ & & - & & & - & & & & & \\
\hline Stevenson \& Kellog, Ltd (1975) & $\bullet$ & $\bullet$ & & & $\bullet$ & & & & & & \\
\hline Knapp et al. (1976) & $\bullet$ & $\bullet$ & & & & & & & $\bullet$ & & \\
\hline Williams-Kuebelbeck \& Associates, Inc. (1976) & $\bullet \cdot$ & $\bullet$ & & - & & & - & & & • & \\
\hline $\begin{array}{l}\text { Leyenda: } 1 \text {-Empleo; } 2 \text {-Sueldos y Salarios; } 3 \text {-No } \\
\text { Generales; } 7 \text { - Compras de Bienes y Servicios; } 8 \\
\text { Directos; y } 11 \text {-Ingresos Fiscales. }\end{array}$ & ; $; 9$ & - & & & & & & & & $\begin{array}{l}\text { astos } \\
\text { tos }\end{array}$ & \\
\hline
\end{tabular}

Fuente: Elaboración propia basado en Kaufmann (1979). 
Tabla 2. Actividades consideradas a la hora de determinar el impacto primario de los puertos según diversos autores.

\begin{tabular}{|c|c|}
\hline$\underline{\text { Autor }}$ & Actividades que se consideran para evaluar los impactos primarios \\
\hline $\begin{array}{l}\text { The Port of Portland } \\
(\mathrm{n} / \mathrm{a})\end{array}$ & $\begin{array}{l}\text { - Actividades Marítimas } \\
\text { - Actividades dependientes del puerto }\end{array}$ \\
\hline Rudy (1961) & $\begin{array}{l}\text { - Cargadores, empresas que desarrollan operaciones en el puerto y suministradores } \\
\text { de servicios en el puerto. } \\
\text { - Industria y comercio dependiente del puerto } \\
\text { - Otras organizaciones } \\
\text { - Consulados } \\
\text { - Asociaciones } \\
\text { - Actividades de agencias locales, estatales y nacionales en relación al puerto }\end{array}$ \\
\hline Roesti et al. (1964) & - Trabajadores directamente empleados en el puerto \\
\hline Conn (1966) & $\begin{array}{l}\text { - Industria de Transporte Marítimo } \\
\text { - Empresas localizadas en el puerto que se consideran dependientes del mismo (en } \\
\text { base a entrevistas) }\end{array}$ \\
\hline Erbuth (1966) & $\begin{array}{l}\text { - Empresas usuarios de Servicio de transporte en el puerto y localizadas en la zona } \\
\text { por razones de la existencia del puerto. }\end{array}$ \\
\hline $\begin{array}{l}\text { Hille \& Suelflow } \\
(1970)\end{array}$ & $\begin{array}{l}\text { - Gasto de buques } \\
\text { - Gasto de las tripulaciones } \\
\text { - Transporte por carretera y ferrocarril al puerto } \\
\text { - Índices de Bancos Industriales y compañías de seguros } \\
\text { - Servicios portuarios } \\
\text { - Empresas dependientes del puerto } \\
\text { - Astilleros } \\
\text { - Gasto del Gobierno en el puerto }\end{array}$ \\
\hline Bragg $(1972,1974)$ & $\begin{array}{l}\text { - Operadores Portuarios } \\
\text { - Refinería }\end{array}$ \\
\hline $\begin{array}{l}\text { Gruen, Gruen \& } \\
\text { Associates (1972) }\end{array}$ & - No se Especifican \\
\hline Schenker (1972) & $\begin{array}{l}\text { - Gasto de los buques en puerto } \\
\text { - Ingresos del puerto y terminales } \\
\text { - Transporte Interior } \\
\text { - Gasto de Tripulaciones } \\
\text { - Servicios Portuarios }\end{array}$ \\
\hline $\begin{array}{l}\text { Oregon Department } \\
\text { of Transportation } \\
(1973)\end{array}$ & - No se Identifican \\
\hline \begin{tabular}{lll|}
$\begin{array}{l}\text { Ryan } \\
(1973)\end{array}$ & $\&$ & Adams \\
\end{tabular} & $\begin{array}{l}\text { - Servicios de Transporte } \\
\text { - Productores }\end{array}$ \\
\hline Dunphy (1974) & $\begin{array}{l}\text { - Empresas de Transporte Marítimo } \\
\text { - Empresas Auxiliares de ámbito Marino } \\
\text { - Empresas de Transporte terrestre } \\
\text { - Organizaciones Públicas y Privadas con relación con el puerto } \\
\text { - Industria marina } \\
\text { - Industria manufacturera } \\
\text { - Industria del sector agrario }\end{array}$ \\
\hline $\begin{array}{l}\text { Whitaker-Mohn } \\
\text { (1974) }\end{array}$ & $\begin{array}{l}\text { - Empresas usuarias del puerto } \\
\text { - Empresas de servicios del puerto } \\
\text { - Empresas de transporte que ofrecen servicios desde o hacia el puerto } \\
\text { - Exportadores. }\end{array}$ \\
\hline Carew (1975) & $\begin{array}{l}\text { - Autoridad Portuaria } \\
\text { - Empresas concesionarios o con autorización para ocupación de zona portuaria }\end{array}$ \\
\hline $\begin{array}{l}\text { Stevenson \& Kellog, } \\
\text { Ltd (1975) }\end{array}$ & $\begin{array}{l}\text { - Empresas directamente relacionadas con el puerto } \\
\text { - Productores y Mayoristas } \\
\text { - Otras Organizaciones Públicas y Privadas }\end{array}$ \\
\hline Knapp et al. (1976) & - Actividades dependientes del puerto (port -dependent activities) ${ }^{1}$ \\
\hline
\end{tabular}




\begin{tabular}{|l|l|}
\hline & $\begin{array}{l}\text { - Actividades relacionadas con el puerto (port related activities) } \\
\text { - Actividades que se desarrollan por la presencia del puerto (harbour related } \\
\text { activities) }^{2}\end{array}$ \\
\hline $\begin{array}{l}\text { Williams-Kuebelbeck } \\
\text { \& Associates, Inc. }\end{array}$ & $\begin{array}{l}\text { - Empresas concesionarias o con autorización para ocupación de zona portuaria } \\
\text { (1976) }\end{array}$ \\
& $\begin{array}{l}\text { - Empresas de prestación de servicios marítimos } \\
\text { - Importadores } \\
\text { - Exportadores }\end{array}$ \\
\hline
\end{tabular}

Nota:

${ }^{1}$ Se considera empresas que realizan actividades dependientes del puerto a aquellas que directamente se encargan de facilitar la manipulación, carga o descarga de mercancías en el puerto.

${ }^{2}$ Se considera empresas que realizan actividades relacionadas con el puerto a aquellas que están relacionadas con la producción, proceso, fabricación o transporte de mercancías que utilizan el puerto (en este estudio se limita a los sectores de manofactura, minería, agricultura y transportes, así como las actividades determinadas entidades y agencias públicas).

${ }^{3}$ Se incluye en este grupo las actividades que sin estar relacionadas con el puerto existen por el hecho de que el puerto existe citando como ejemplo actividades en el entorno de la carretera que da acceso al mismo.

Fuente: Elaboración propia basado en Kaufmann (1979).

Tabla 3. Metodología para la determinación de los impactos secundarios o estimación de ahorros en el coste de transporte por razón de la existencia del puerto.

\begin{tabular}{|c|c|c|}
\hline$\underline{\text { Autor }}$ & $\begin{array}{l}\text { Metodología de determinación de los } \\
\text { impactos secundarios }\end{array}$ & $\begin{array}{l}\text { Estimación de ahorros en coste } \\
\text { de transporte }\end{array}$ \\
\hline The Port of Portland (n/a) & No se calculan & No se calculan \\
\hline Rudy (1961) & No se calculan & No se calculan \\
\hline Roesti et al. (1964) & $\begin{array}{l}\text { Estimaciones basadas en EIEP previo (Puerto de } \\
\text { Stockton, California) }\end{array}$ & $\begin{array}{l}\text { Estimación de los ahorros } \\
\text { unitarios por las mercancías } \\
\text { embarcadas / desembarcadas en el } \\
\text { puerto }\end{array}$ \\
\hline Conn (1966) & $\begin{array}{l}\text { Estimación Basada en la experiencia del autor y } \\
\text { otros EIEP }\end{array}$ & $\begin{array}{l}\text { Diferencial entre el coste del } \\
\text { transporte marítimo y su } \\
\text { alternativa (transporte por } \\
\text { ferrocarril y barcaza). }\end{array}$ \\
\hline Erbuth (1966) & $\begin{array}{l}\text { Estimaciones basadas en coeficientes } \\
\text { multiplicadores de comunidades de similar } \\
\text { características. }\end{array}$ & No se calculan \\
\hline Hille \& Suelflow (1970) & Ninguno & No se calculan \\
\hline Bragg (1972) & $\begin{array}{l}\text { Modelo Input-Output y multiplicadores del } \\
\text { sector del petróleo y refinería }\end{array}$ & No se calculan \\
\hline $\begin{array}{lcc}\text { Gruen, } & \text { Gruen } & \& \\
\text { Associates } & (1972)\end{array}$ & $\begin{array}{l}\text { Multiplicador Input-Output ajustado de otro } \\
\text { estudio previo (se cita un EIEP en el puerto de } \\
\text { Seattle de } 1969 \text { pero no se indica cual) }\end{array}$ & No se calculan \\
\hline Schenker (1972) & $\begin{array}{l}\text { Estimaciones basadas en estudios realizados en } \\
\text { regiones cercanas (Estudios realizados por el } \\
\text { Southeastern Wisconsin Regional Planning } \\
\text { Commissioning para esta región) }\end{array}$ & No se calculan \\
\hline $\begin{array}{l}\text { Oregon Department of } \\
\text { Transportation (1973) }\end{array}$ & $\begin{array}{l}\text { Análisis basado en teoría económica: } \\
\text { determinación de la base económica siguiendo } \\
\text { una aproximación de requerimientos mínimos } \\
\text { (Ullman \& Dacey, 1960) }\end{array}$ & $\begin{array}{l}\text { Coste mínimo del transporte de } \\
\text { las mercancías movidas en el } \\
\text { estado menos el coste mínimo de } \\
\text { transporte sin la presencia del } \\
\text { puerto }\end{array}$ \\
\hline Ryan y Adams (1973) & $\begin{array}{l}\text { Análisis basado en teoría económica (sin aportar } \\
\text { datos sobe los sectores utilizados) }\end{array}$ & No se calculan \\
\hline Brag (1974) & No se calculan & No se calculan \\
\hline Dunphy (1974) & $\begin{array}{l}\text { Análisis basado en teoría económica: } \\
\text { determinación de la base económica tomando }\end{array}$ & No se calculan \\
\hline
\end{tabular}




\begin{tabular}{|c|c|c|}
\hline & $\begin{array}{l}\text { hipótesis ad-hoc y metodología de Coeficientes } \\
\text { de Localización }\end{array}$ & \\
\hline Whitaker-Mohn (1974) & $\begin{array}{l}\text { Estimaciones basadas en EIEP previo } \\
\text { (Coeficiente Multiplicador ‘3.0’ usado en el } \\
\text { puerto de Stockton) }\end{array}$ & No se calculan \\
\hline Carew (1975) & $\begin{array}{l}\text { Estimación basada en la experiencia del autor en } \\
\text { EIEP y un estudio previo el área metropolitana } \\
\text { del puerto (California) }\end{array}$ & No se calculan \\
\hline $\begin{array}{l}\text { Stevenson \& Kellog, Ltd } \\
(1975)\end{array}$ & Análisis basado en TIO & No se calculan \\
\hline Knapp et al. (1976) & $\begin{array}{l}\text { Estimaciones basadas en estudios realizados en } \\
\text { estudios de otra naturaleza (estudios sobre } \\
\text { crecimiento del empleo en el área de estudio) }\end{array}$ & No se calculan \\
\hline $\begin{array}{l}\text { Williams-Kuebelbeck \& } \\
\text { Associates, Inc. (1976) }\end{array}$ & $\begin{array}{l}\text { Enfoque desde la perspectiva de gastos e } \\
\text { ingresos }\end{array}$ & No se calculan \\
\hline
\end{tabular}

Fuente: Elaboración propia basado en Kaufmann (1979).

\subsection{El Port Economic Impact Kit.}

Recogiendo todos estos estudios e inquietudes, Arthur D. Little, Inc. (1979) realiza un trabajo para la U.S Maritime Administration (MARAD) que, bajo el nombre "Port Economic Impact Kit", trataba de presentar una estandarización metodológica sobre la que se basasen estudios posteriores que permitiese su comparación. Dicha metodología resulta una adaptación ad hoc al caso portuario del clásico modelo TIO y desde su lanzamiento ha visto sucesivas adaptaciones como la de MARAD (1982), Temple, Barker y Sloane Inc. et al. (1985), MARAD (1995) y la lanzada en el año 2000 para ser implementada sobre un PC y que comprende un análisis de 30 sectores incluyendo modelización de las operaciones relativas a todo tipo de tráficos (contenedores, graneles líquidos, sólidos, carga general, automóviles, cruceros, pasajeros, ferries...) y obras de construcción y dragados (MARAD, 2000).

En esencia el Port Economic Impact Kit tal y como se presentó en 1979 establecía una metodología para determiner, por un lado, los impactos directos de las actividades directamente dependientes del puerto y lo que denominaba estimación de efectos secundarios. Para los primeros establecía la necesidad de hacer estudios de campo específicos en lo que en una primera fase determinar los usuarios y prestatarios de servicio del puerto, determinando el grado de dependencia de los mismos del puerto (ofrecía incluso los modelos a encuestas a implementar) y, en un segundo, proceder a tomar datos de su actividad. Para el establecimiento de efectos secundarios enunciaba que tres métodos eran posibles: i) la utilización de multiplicadores de base económica, ii) los multiplicadores de comercio interregional y, iii) las tablas de input-output.

La utilización de multiplicadores de base económica se basa en la consideración de dos grupos dentro de la región sometida a estudio. Una primera con las empresas que producen bienes y servicios que son exportados fuera de la región y una segunda con las empresas que realizan sólo un consumo local. Se trata éste de un procedimiento simple y que no requiere de una extensiva recopilación de datos o una profunda formación económica.

La utilización de multiplicadores de comercio interregional en EIEP fueron desarrollados partiendo de la teoría de determinación de ingresos Keynesiana (Keynes, 1936) y está basada en la relación entre los ingresos y las componentes que lo constituyen (consumo, inversiones, gastos gubernamentales, importaciones y exportaciones). Resulta un método un poco más complejo que el de multiplicadores de base económica, pero, a su vez, es más simple que el de TIO con el que presenta ciertas similitudes pero que, a diferencia de éste, no proporciona disgregación sectorial que permita analizar la relación entre diferentes sectores. 
Por último, la premisa básica de la metodología TIO es establecer las relaciones por las que cada industria vende su producción a otras industrias o consumidores finales y en contrapartida compra bienes y servicios de otras industrias o productores primarios (capital o mano de obra). Siendo así, el rendimiento de cada sector se ve afectada por la demanda final y las interrelaciones sectoriales (Leontief, 1966). Desde el punto de vista teórico, la disgregación sectorial es la que permite una mejor y más acertada aplicación al caso portuario si bien, ha de considerarse como un método costoso en cuanto a la confección de dichas tablas y debe tomarse como referencia las publicaciones de organismos oficiales encargadas de recopilar estos datos.

Los autores enfatizaban en la necesidad de caracterizar con rigor los efectos de la empresa dependiente puesto que posibles errores en este cálculo se verían amplificados al calcular los efectos de orden secundario.

\subsection{Depuración y mejora de los EIEP: desarrollos en la década de los 80 y 90.}

Continuando con el desarrollo metodológico asociado a los EIEP, Davis (1983) vuelve a poner el foco sobre el objetivo de este tipo de estudios indicando que en general pueden englobarse en aquellos que tratan de evaluar los impactos de un puerto existente y aquellos otros que buscan determinar el impacto que producen cambios incrementales en la actividad portuaria y en sus servicios.

Para este autor, hasta la fecha la mayoría de los estudios se englobaban dentro de este primer grupo y respondían, tal y como otros autores ya habían señalado, a proporcionar una herramienta de "relaciones públicas" para los gestores portuarios en un marco más político que técnico-económico.

Señala que tres son las debilidades de los estudios desarrollados hasta la fecha y que cuatro metodologías han sido las empleadas en estos estudios.

En lo que se refiere a la metodología señala cuatro alternativas: i) aplicación de multiplicadores deducidos de estudios previos, ii) estudios de base económica basados en dividir la economía regional en un mercado "local" que no tiene relaciones comerciales fuera de la región y un mercado "exportador" que sí lo tiene, iii) análisis de ingresos y gastos o análisis de multiplicador keyniesiano y iv) metodología TIO o método Leontief de coeficientes técnicos constantes. La adopción de uno u otros métodos debiera depender de los recursos disponibles siendo los más económicos los primeros y los más costosos los últimos; pero también los más apropiados los últimos pues van superando sucesivas debilidades de los modelos anteriores. En la Tabla 4 se presenta un resumen de la metodología usada por algunos autores relevantes mencionados con anterioridad.

En cuanto a los defectos Davis (1983) señala los siguientes: i) inadecuada determinación de qué son impactos primarios del puerto, ii) fallos metodológicos en la forma de evaluar los efectos secundarios y iii) el incorrecto uso de los resultados de los estudios para evaluar los cambios en el nivel o volumen de las actividades portuarias.

Para este autor, la debilidad inherente a la deficiente evaluación de efectos directos de las actividades portuarias proviene de que en muchas TIO utilizadas no existía un elemento (fila y columna) que incluyese específicamente el sector portuario lo que obligaba a realizar asunciones sobre qué sectores debían ser considerados en los estudios. Esto, no se hacía siempre de una manera homogénea (distintos estudios utilizaban distintas filas y columnas) lo que se justificaba porque la estructura de relaciones entre sectores puede ser cambiante en cada región. Para solventar esta carencia, la Autoridad Portuaria de Nueva York - New Jersey había promovido una reformulación de las tablas nacionales input - output incluyendo como sector específico la "industria portuaria" (MARAD, 1978). 
Tabla 4. Métodos y autores para la realización de EIEP.

\begin{tabular}{|c|c|}
\hline Método & Autor \\
\hline $\begin{array}{l}\text { Multiplicadores y otros datos } \\
\text { deducidos de estudios previos }\end{array}$ & $\begin{array}{l}\text { Anderson (1964) } \\
\text { Conn et al. (1966) } \\
\text { Roestim et al. (1964) } \\
\text { Gruen, Gruen \& Associates (1972) } \\
\text { Condor \& Tynerson (1972) } \\
\text { Dunphy \& Chang (1973) } \\
\text { Ryan \& Adams (1973) } \\
\text { Pender \& Wilder (1974) } \\
\text { Carew (1975) } \\
\text { Knapp et al. (1976) }\end{array}$ \\
\hline $\begin{array}{l}\text { Análisis de ingresos y gastos } \\
\text { (multiplicador Keyniesiano) }\end{array}$ & $\begin{array}{l}\text { Williams-Kuebelbeck \& Associates Inc. (1976) } \\
\text { McMullen \& Associates Inc. (1978) } \\
\text { Booz-Allen \& Hamilton Inc. (1982) }\end{array}$ \\
\hline Análisis de Base Económica & $\begin{array}{l}\text { Anderson (1964) } \\
\text { Erbguth (1966) } \\
\text { Oregon Department of Transportation (1973) } \\
\text { Dunphy \& Chang (1973) } \\
\text { Ryan \& Adams (1973) }\end{array}$ \\
\hline Meotodología TIO & $\begin{array}{l}\text { Anderson (1964) } \\
\text { Armenakis et al. (1970) } \\
\text { Port of Seattle Commision (1971) } \\
\text { Bragg (1974) } \\
\text { Carew (1975) } \\
\text { Hille (1975) } \\
\text { Mulligan \& Collins (1975) } \\
\text { Stevenson \& Kellog, Ltd (1975) } \\
\text { Economics Research Associates (1976) } \\
\text { MARAD (1978) } \\
\text { Williams-Kuebelbeck \& Associattes, Inc. (1978) } \\
\text { Standford Research Institute (1979) } \\
\text { Beeman Associattes \& Mannalytcis (1980) } \\
\text { Temple y Barker \& Stone Inc. (1982) } \\
\end{array}$ \\
\hline
\end{tabular}

Fuente: Elaboración propia basado en Kaufmann (1979) y Davis (1983).

En lo que se refería a la forma de evaluar los efectos secundarios, el autor señala las principales deficiencias de cada una de las metodologías anteriores para concluir que, el más acertado resulta el análisis de las TIO porque es capaz de identificar diferentes multiplicadores para cada uno de los sectores económicos del modelo algo que no es posible con los modelos de base económica o de ingresos y gastos.

Por lo que se refiere a los defectos derivados de la evaluación de los cambios en el volumen o nivel de las actividades portuarias cita como ejemplo: i) que los efectos introducidos por los multiplicadores se miden en términos medios y no en marginales cuando pequeños cambios en la actividad portuaria deberían tener en consideración estos últimos y, ii) que la propia formulación de coeficientes técnicos constantes no es capaz de capturar cambios tecnológicos y que en estudios de largo plazo este tipo de avances deberían inducir cambios en los coeficientes como respuesta de los factores económicos a los mismos y la acomodación de éstos en las relaciones entre sectores.

Otros autores que han ayudado a depurar las técnicas de EIEP basados en modelos de TIO han sido Yochum y Arwal $(1987,1988)$. Estos investigadores presentan un estudio con el que tratan de solventar carencias de estudios anteriores en lo que se refiere a simplificaciones para evaluar los impactos directos y presentan una nueva técnica basada en TIO para abordar problemas que se han puesto de manifiesto en la estimación de impactos secundarios. Aplican su modelo en el EIEP desarrollado en Port of Hampton Roads (Virigina, USA) con datos de 1984. 
Estos autores propusieron una nueva aproximación para la elaboración de EIEP siendo su principal contribución la adopción de un criterio homogéneo y una metodología común para calcular el impacto primario de los puertos definiendo previamente el grado de dependencia que las empresas de su hinterland tienen en relación a esta infraestructura. Este enfoque será posteriormente seguido por multitud de autores superando la falta de investigación que sobre el particular había sido puesto de manifiesto por Davis (1983).

En lo que se refiere al criterio, estos autores distinguen entre aquellas empresas que son necesarias para el funcionamiento del propio puerto ("required industries"), las que han elegido su localización en consideración de la presencia del puerto y la influencia que en sus costes de transporte y logísticos esto tiene ("attracted industries") y las que se benefician de la presencia del puerto y han podido expandir su mercado con importaciones o exportaciones a través de él pero cuya decisión de localización no es estrictamente dependiente de la existencia del puerto ("induced industries").

En lo que se refiere al segundo grupo de empresas, de no existir el puerto éstas se verían tan severamente afectadas que se plantearían el cierre o desplazar sus actividades a otras localizaciones pudiendo ser consideradas junto con el primer grupo como empresas íntegramente generadoras de impacto primario y, por tanto, empresas dependientes del puerto. En el caso del último grupo, sólo un porcentaje de las actividades de estas industrias puede ser atribuido al puerto, siendo necesario estimar cuál es el porcentaje adecuado puesto que de no existir el puerto estas empresas ajustarían sus actividades, pero no necesariamente cerrarían o se deslocalizarían.

Por ello y desde el punto de vista metodológico es necesario a juicio de los autores realizar una campaña intensiva de campo con encuestas a las empresas asentadas en el hinterland del puerto determinando el porcentaje de sus empleados que están directamente relacionados con las actividades del mismo. En ausencia de datos de campo, estos autores proporcionan una tabla de referencia con el tipo de actividad y el porcentaje de ocupación extraído de su caso práctico.

Adicionalmente estos autores identifican algunas constricciones para identificar los impactos secundarios producidos en el puerto que tiene que ver con que los modelos TIO casi nunca consideran la industria portuaria como un elemento específico dentro del mix interindustrial, por lo que es necesario establecer hipótesis sobre que otras industrias pueden considerarse dentro de este capítulo y en qué proporción.

Aunque es cierto que esta carencia de datos podría ser parcialmente cubierta con las encuestas a las empresas si éstas proporcionasen datos sobre el volumen de actividades y facturación con las empresas que hemos identificado arriba (en cualquiera de sus categorías); no es menos cierto que muchas empresas no proporcionan dichos datos en aras de un lógico celo de protección y secreto comercial.

Para solventar este problema los autores desglosan los ingresos promedio relacionados con el puerto en categorías de gastos según las estimaciones incluidas en la Encuesta de Gastos del consumidor del Departamento de Trabajo de Estados Unidos y los gastos se procesan a través de las tablas I-O de cada sector industrial particular. Citan como ejemplo cómo los gastos promedio para una categoría de ingresos específica en las compras al por menor se procesan a través del sector de comercio minorista del modelo.

Para superar la barrera metodológica relacionada con la inexistencia de filas y columnas específicas de industria portuaria algunos autores habían propuesto modelos alternativos como los modelos de control de grupo de Isserman y Merrifield (1982) que han tenido poco recorrido científico. Posteriormente otros investigadores proponen alternativas como la utilización de modelos basados en encuestas de los propios Yochum y Agarwal (1988) o Gripaios y Gripaios (1995), los modelos de oferta - demanda de de Salvo (1994), los modelos de elasticidad de los precios de demanda (de Salvo \& Fuller, 1995), los modelos usando coeficientes de localización de Musso et al. (2000), o los de equilibrio general con consideración espacial de Bichou (2007) o 
Haddad et al. (2010) sobre los que más tarde volveremos para no romper la descripción cronológica adoptada en este artículo.

Autores como Randall (1988) amplían el foco de las actividades a desarrollar por las Autoridades Portuarias, investigando el efecto derivado de inversiones no estrictamente portuarias (desarrollo de suelo, construcción de infraestructuras colaterales como accesos, puentes o túneles) y las consecuencias positivas tanto para el desarrollo del puerto como para el fortalecimiento de las relaciones entre el puerto y su comunidad local.

La literatura académica sobre este tema siguió creciendo a finales de la década de los $80 \mathrm{y}$ comienzos de la 90 con muchas realizaciones entre las que cabría destacar las de Behan (1988), de Salvo y Fuller (1988), Conway et al. (1989), Warf y Cox (1989), Braun (1990), Opuku, (1990), Pinfold (1991), de Salvo (1994), Gripaios y Gripaios (1995) y de Salvo y Fuller (1995).

Estos últimos autores, analizando el rol de la elasticidad del precio del lado de la demanda en los impactos económicos llegaban a la conclusión de que utilizando una aproximación estándar como la que se venía implementando (refiriéndose específicamente al Port Economic Impact Kid de MARAD) los impactos eran sobreestimados ya que se asume implícitamente que todas las actividades de importación y exportación de una región se hacen cero en el caso de cese de actividad del puerto por el que se transportan dichas mercancías algo que, obviamente, no es cierto con carácter general. Para ello realizan un estudio sobre el puerto de Tampa (Florida, USA) y ofrecen una metodología alternativa con la que contrastar los resultados y cuantificar la posible sobre-estimación (que en su caso experimental evalúan en rangos del 93\% para las exportaciones y del $96 \%$ para las importaciones).

Dicha metodología que se resume en de Salvo y Fuller (1995) pero que se describe con más detenimiento en de Salvo y Fuller (1988) solo aborda el cálculo del impacto directo de los puertos y no incorpora avances en lo que se refiere a los efectos indirectos para los que se sigue proponiendo la utilización de metodología TIO para estimar los coeficientes multiplicadores. El método parte de la recopilación de los datos de los precios medios y volúmenes de mercancías declarados ante el Departamento de Comercio por las empresas de la zona de influencia del puerto, que se comparan con el volumen de dichas mercancías que realmente pasan por el puerto (utilizando los datos de la Autoridad Portuaria) y a los que se aplican unos coeficientes de elasticidad de la demanda que se toman de diferentes fuentes bibliográficas. Con ello se calcula la cuota de mercado de cada mercancía y la cuota de mercado "ajustada" (afectada por el coeficiente de elasticidad) para lo que se utilizan curvas de estimación precio - demanda de tipo lineal y con las que se calcula: i) cuál es la sensibilidad al cambio de precio de las mercancías para aquellos compradores de dichas mercancías que denomina "no impactados", ii) cuál es la sensibilidad de las importaciones locales a cambios en el precio en la zona "impactada". Considerando la cuota de mercado ajustada por la elasticidad y aplicándoles un coste unitario de transporte marítimo (fletes, costes portuarios, costes aduaneros) y de transporte terrestre (incluyendo el propio transporte y los seguros) estimados por los autores en base a datos reales calcula cuál es el impacto directo en el caso de cierre del puerto que compara con los resultados que se hubieran obtenido utilizando el Port Economic Impact Kit de MARAD.

\subsection{El periodo de expansión. EIEP en el siglo XXI. Aparición de métodos alternativos.}

El hecho es que, a lo largo de los años, el número de realizaciones no ha dejado crecer pudiendo encontrar, como ya indicaban Villaverde-Castro y Coto Millán (1997) dos tipos de trabajos. Aquellos de naturaleza más académica o científica promovidos desde universidades o centros de investigación y aquellos de carácter más comercial realizadas por empresas consultoras para diferentes organismos portuarios.

Ejemplo destacado de estos últimos tendríamos a Martin Associates LLC quienes ya en el año 2008 habían reportado haber realizado más de 300 EIEP (Martin Associates, 2008) y que a la fecha hacen extensivo a un conjunto de 73 puertos fundamentalmente de Estados Unidos y Canadá (Martin Associates, 2017) y sobre los que realizan periódicas actualizaciones, lo que 
permitirá futuras líneas de investigación centradas en determinar el carácter evolutivo de los impactos portuarios en las regiones (como ejemplo citaríamos las actualizaciones realizadas sobre el puerto de Portland de Martin Associattes 2007a, 2012, 2016a).

Rodríguez-Dapena (2005) proporciona una revisión de la metodología para los EIEP y se proporciona una interesante comparativa de los estudios realizados hasta la fecha en un conjunto de 21 Puertos del Sistema Portuario Español, discriminando entre los efectos de la industria portuaria y la industria dependiente y discutiendo cuestiones metodológicas y la utilización de herramientas de predicción o simulación en el marco de la evaluación económica del papel de los puertos. Para este autor el enfoque de los EIEP podía limitarse a un análisis estático de un puerto existente o centrarse en el análisis de un proyecto de nuevo puerto o expansión de uno existente con técnicas de previsión o simulación (ex ante o ex post) en el que se analicen escenarios de ampliación, paralización total o parcial del puerto o cambios en la oferta portuaria (p.e. impactos de políticas tarifarias).

Bichou (2007) ofrece una nueva clasificación de la tipología de estudios que han venido desarrollándose. Así, a los ya citados estudios basados en TIO, incluye los modelos de equilibrio general o CGE (computable general equilibrium) y modelos de gravedad (gravity models).

Los CGE (Arrow \& Debreu, 1954) son métodos de análisis cuantitativo que combinan teoría económica con datos económicos reales y que están especialmente indicados para evaluar el impacto económico introducido por la adopción de reformas e implantación de diferentes medidas políticas. En ambiente portuario han sido utilizados en puertos japoneses (Doi et al. 2002), brasileños (Haddad et al., 2010), de Taiwan (Chen et al. 2011), o en aplicaciones colaterales como el análisis del impacto regional de un corredor ferroviario con origen en el puerto de Algeciras (Cardenete-Flores \& López-Cabaco, 2016).

Los modelos de gravedad en comercio internacional (Tinbergen, 1962) tratan de predecir los movimientos comerciales basándose en los tamaños de las economías (utilizando generalmente el PIB como indicador) y la distancia entre las mismas. Presentan como ventaja respecto a los modelos TIO o CGE que son menos intensivos en lo que se refiere a los requerimientos de datos para componer el modelo (Costacurta de Sá Porto, 2010) siendo de más rápida y fácil aplicación. En ambientes portuarios se ha utilizado para proporcionar una estimación del tamaño del hinterland de una terminal de contenedores realizando una partición espacial del territorio (Yang, 2002; Yang et al., 2011, 2012), el ámbito territorial asociada a una línea de cabotaje de contenedores (Vido, 2004; Sheng et al., 2013), o el rol de los puertos en las exportaciones y flujos comerciales de sus regiones o estados como los estudios de Costacurta de Sá Porto (2010) sobre el sistema portuario Brasileño o los de Onyemechi et al. (2014) para analizar el caso nigeriano.

Autores posteriores como Pallis et al. (2009a, 2009b, 2011) y Coto-Millán (2010b) también ofrecen criterios de clasificación metodológica de los EIEP.

Por último, de la Peña (2018a) indica como desde la primera década de los 2000 una nueva escuela de EIEP se ha desarrollado en China utilizando como técnica de análisis la modelización dinámica, paradigma de programación presentado originalmente por J.W. Forrester y desarrollado con posterioridad por sus discípulos del MIT. Entre autores de esta corriente, con una clara componente ambientalista, destacan Han (2006), Cui (2007), Yang (2007), Cao (2009), Zhao (2009), Liu et al. (2010), Zhang (2010), Wang (2010), Xiaodong et al. (2010), Fu (2011a, 2011b), Dai (2012), Xing (2012), Gao (2012), Yang (2012), Li y Wang (2012), Wang et al. (2013) y Yu et al. (2014).

Todos estos autores encuentran un campo de investigación apropiado por el crecimiento exacerbado que ha experimentado China en los últimos años con el consiguiente incremento de sus exportaciones y las grandes tensiones introducidas en su sistema portuario y con ello en el medio ambiente y ciudades/regiones en que se han desarrollado sus obras de expansión. Con carácter general, desarrollan los diagramas causales en los que se establecen las relaciones entre 
distintas variables económicas y ambientales que modelizan con la ayuda de distinto software de Simulación Dinámica (SD).

Sin necesidad de describir con detalle los estudios de todos estos autores (cuya descripción detallada se encuentra en de la Peña (2018a)) podemos tomar como ejemplos representativos a Zhao (2009) y Liu et al. (2010).

El primero trata de analizar el desarrollo portuario bajo un balance global de los efectos que produce en el desarrollo urbano. Sin negar los efectos positivos de las actividades portuarias, éstas se ponen en contrapunto con los fenómenos de ocupación de la línea de costa, la contaminación inducida por sus actividades y la posible alteración del equilibrio ecológico de la zona donde se asientan. El enfoque es, por tanto, el de restar a los efectos positivos producidos por el puerto, las externalidades que éste genera, de tal manera que se consiga una "contribución verde" del puerto a la ciudad.

Los segundos siguen avanzando en el estudio analítico de "ciudades portuarias" bajo aproximaciones SD. Sus estudios son más en términos de los efectos que induce el puerto en el entorno (como consumidor de recursos o introductor de efectos ambientales) que del propio análisis de las operaciones portuarias, aceptando los propios autores que su enfoque está basado en un "concepto verde" que no ocultan ni en el propio título del artículo y que venían siendo tratados por autores anteriores como Han (2006) quien empieza a analizar de una manera cuantitativa mediante modelización SD las externalidades que producen los puertos, o Zhao (2009) quien utiliza la misma terminología de "puerto verde". Consideran como aspectos relevantes del medio: el desarrollo económico (aspectos positivos que miden en forma de la contribución del puerto al PIB de su zona de influencia) y el consumo de recursos o la contaminación inducida (estos dos últimos, aspectos negativos que evalúan por ejemplo en los insumos de agua o consumos energéticos de las industrias relacionadas con el puerto) y lo aplican como caso experimental concreto al puerto de Shangai (China). Los autores desarrollan para este puerto escenarios interactivos que simulan hasta el año 2020 y en los que consideran conjuntamente medidas de restructuración industrial, realización de inversiones portuarias, ahorros energéticos y reducción de emisiones. Determinan finalmente como los sistemas SD pueden ser una herramienta eficaz para la toma de decisiones evaluando de una manera global las consecuencias ambientales de las decisiones tomadas como palanca para proporcionar un desarrollo sostenible.

\section{Utilidad, justificación y crítica de los EIEP.}

La utilidad de los EIEP como herramienta con la que resaltar el efecto multiplicador del puerto y los beneficios de sus actividades ha sido un elemento puesto de manifiesto y discutido en muchos estudios ya citados (Waters, 1977; Chang, 1978; Suykens, 1989; Gripaios \& Gripaios, 1995; Verbeke \& Debisschop, 1996).

Sin embargo, la utilización de estos estudios con ese carácter exclusivo dentro de la esfera política no ha dejado de estar exenta de polémica y crítica científica. Entre los autores que han abordado los estudios de impacto económico bajo este tamiz crítico advirtiendo a cerca del riesgo de sobreestimación de resultados podríamos destacar a Davis (1983), Gripaios y Gripaios (1995), Verbeke y Debisschop (1996), Gripaios (1999), Crompton (2006), Musso et al. (2006), Musso et al. (2011) y Danielis y Gregori $(2012 ; 2013)$.

Probablemente el más crítico de todos ellos sea Crompton (2006) quien a través de una serie de ejemplos prácticos explicita los abusos que se hacen de este tipo de estudios en el caso concreto de estudios de repercusión económica de eventos y competiciones deportivas pero que bien pueden ser extrapolables a ambientes portuarios. Entre los defectos que este autor indica encontraríamos los siguientes: 
1. Los resultados de los estudios pueden venir viciados ex ante por el interés de su promotor (usualmente la Autoridad Portuaria en el caso de los EIEP) en magnificar los efectos positivos de la actividad que promueve.

2. Consideración como un impacto de la actividad el consumo de los residentes locales, cuando de no realizarse la actividad elegirían una actividad alternativa dentro de esa misma comunidad (y, por tanto, no generando ningún valor real sino desplazamiento de los flujos). En el caso de los EIEP éste sería el efecto puesto de manifiesto por de Salvo y Füller (1988, 1995) quienes con el modelo de elasticidad antes indicado explican cómo es errónea la hipótesis de muchos autores que consideran que la actividad económica asociada a una determinada mercancía que utiliza el puerto se hace cero en el caso de que el puerto deje de existir.

3. Inapropiada agregación geográfica. Esto puede ocurrir cuando la escala geográfica del área sometida a estudio varía y se agregan los impactos de dos estudios como simple suma de los impactos individuales de cada uno de estos estudios, ignorando los solapes que pueden existir entre ambos modelos (de tal manera que puedan producirse problemas de doble contabilización). Este efecto también se puede producir al tomar separadamente dos EIEP que pueden considerar en sus estudios demanda la misma mercancía, siendo especialmente aplicable al caso de los tráficos desviados que describimos en el apartado $4 \mathrm{o}$ tal y como exponen Goss (1990), Musso et al. (2011) o Danielis y Gregori (2012) cuando las rentas generadas por determinada actividad portuaria se computan a su región cuando en realidad se "drenan" a otras más lejanas.

4. Efectos "time-switchers" y "casual": contabilización como impactos creados por una actividad, impactos que en realidad se generan por otra distinta. Pone como ejemplo cómo en determinadas ocasiones una persona puede acudir circunstancialmente a un evento deportivo porque está de visita a una ciudad (o hace coincidir una visita ya decidida con el evento aprovechando su celebración) pero que el motivo de la visita a la ciudad no es el evento sino otro distinto. De no existir el evento, esta persona hubiera acudido de igual modo a la ciudad, pero escogido otro momento y/o actividad de ocio (con lo que no existe generación de valor por la celebración de dicha actividad). En el caso de los EIEP esta errónea contabilización también es posible en el caso de aquellas mercancías que, de no existir el puerto, seguirían estando presentes en la región, pero utilizarían otros modos de transporte $\mathrm{u}$ otro puerto alternativo.

5. Abuso en el uso de multiplicadores: i) inclusión del consumo de residentes locales en el evento (que en realidad no añade nuevo valor en el sistema) algo que ocurre en los EIEP si tenemos en cuenta que parte de los trabajadores que se consideran dependientes del puerto no abandonarían la zona en el caso de que el puerto no existiese (pudiendo encontrar un puesto de trabajo en una actividad que ha sido desplazada por la presencia del mismo), ii) enfatización y mal uso del multiplicador de ventas (que produce un mayor efecto económico que el multiplicador de ingresos pero es menos apropiado que este último para valorar el impacto económico real sobre los residentes de la zona); iii) mal uso del multiplicador de empleo (relacionando efectos como la asunción de que todos los puestos de trabajo están empleados al $100 \%$ y por tanto que cada hora adicional de trabajo producida por el evento supone un nuevo trabajador; con la contabilización de las horas de empleo parcial con la misma consideración que los de tiempo completo ignorando el hecho de que un evento de estas características puede no incrementar el número de horas totales de trabajo sino desplazar ciertas tareas que hacen empleados fijos para acomodarse al pico de actividad que introduce el evento, de tal manera que no sea necesario contratar a un trabajador eventual siendo este trabajo asumido por alguien que ya trabaja; o por la asunción de que todas las horas adicionales de trabajo serán asumidas por residentes locales y no por empleados que vengan de áreas externas) (efecto que también puede "contaminar" los EIEP y para lo que es necesario determinar cuál es el grado de dependencia de un empleo a la existencia del puerto tal y como hemos visto que abordan Yochum y Agarwal (1987)) y, iv) fallo en la contabilización de los volúmenes de ventas que siendo adquiridos en la región donde se 
celebra el evento son producidos total o parcialmente en otra región pero se contabilizan exclusivamente en la primera ("capture rates") (efecto que de igual manera se puede dar en un EIEP si se incorporan como impactos positivos asociados a la actividad portuaria productos y servicios que en realidad provienen de otra región).

6. No consideración de los costes de oportunidad, es decir, que otros efectos positivos se hubiesen producido de no derivarse los recursos a la actividad que se evalúa (sino a otra de distinta naturaleza o simplemente reteniéndoles sin utilización) y necesidad de "netear" los efectos positivos de una determinada actividad con la de sus posibles sustitutas. De igual modo este efecto también está presente en los EIEP, en tanto que la actividad portuaria puede desplazar potencialmente otras actividades (ocio y turismo, pesca, desarrollo inmobiliario...) cuyos posibles efectos positivos no se evalúan en los EIEP y se descuentan del impacto generado por el puerto.

7. No consideración de los costes de desplazamiento que se producen cuando, por ejemplo, un visitante a un evento ocupa una plaza hotelera que hubiese sido utilizada por otro visitante a la ciudad pero que no hubiera acudido al mismo. Este efecto también puede darse en los EIEP cuando determinados tráficos movidos a través de un puerto y considerados en el estudio desplazan a otros tráficos complementarios o sustitutorios que no utilizarían el puerto dentro de su logística.

8. No consideración de las externalidades y costes soportados por las comunidades locales con el desarrollo de la actividad valorando sólo los efectos positivos. De igual modo, las externalidades asociadas a impactos ambientales o situaciones de congestión no suelen ser incorporados en los EIEP algo que, como veremos en el apartado 4, puede abordarse a través de metodologías Coste - Beneficio.

9. Inadecuada expansión del alcance del proyecto o actividad que se somete a análisis, añadiendo al impacto de una actividad concreta los de otras actividades que en realidad no están relacionados con la misma y que se incluyen en el estudio para hacer ver un impacto más positivo que el realmente producido. Los EIEP pueden igualmente adolecer de esta deficiencia si, por ejemplo, se considera determinada actividad o empresa dentro de los efectos primarios cuando realmente dicha actividad no es dependiente del puerto y se magnifica aguas arriba cuando además se incorpora un coeficiente multiplicador para estimar los impactos secundarios.

10. Exageración de la demanda (número de visitantes en el ejemplo usado por el autor, o número de toneladas movidas por el puerto en un ejemplo portuario).

11. Incorporación en los estudios del impacto estimado que produce el excedente del consumidor (p.e. lo que un usuario estaría dispuesto a gastarse en un evento), que puede legítimamente usarse como indicador del valor económico de una actividad, pero que no refleja el impacto real en la economía de la misma (gasto real que el visitante realiza). Esta desviación se hace presente en los EIEP a través de las propias hipótesis de la metodología TIO y el efecto de los coeficientes multiplicadores.

Musso et al. (2011) defienden la utilización de los EIEP en el marco de los procesos de aprobación de las expansiones portuarias y lo justifican sobre la necesidad de contraponer los efectos positivos y negativos de estas inversiones y en el que el desarrollo de estas infraestructuras se completa como un trade-off entre el efecto positivo en términos de empleo e ingresos locales y la oposición local que suele acompañar a estos desarrollos. Al tiempo, estos autores indican como los impactos económicos de los puertos están drenándose y ciertos efectos locales se están trasladando a una mayor escala (efectos regionales, nacionales o supranacionales) toda vez que los usuarios del puerto extraen las rentas producidas en los puertos en los que operan y las trasladan a localidades más lejanos produciéndose una auténtica reorganización de los costes y beneficios de los puertos en un ambiente dinámico de evolución del hinterland portuario. Estos 
autores proponen que se evalúen con mayor detenimiento las externalidades que produce el puerto y reclaman un estudio más detallado de la distribución territorial de efectos.

Musso et al. (2006) ya habían tratado este asunto desde esta perspectiva advirtiendo del riesgo de que las comunidades locales valorasen sus puertos como menos "deseables" al sufrir los costes de su desarrollo pero no retener los beneficios del mismo, enfoque anunciado una década antes por Goss (1990) al detectar cuatro riesgos que podían hacer que un desarrollo portuario no fuese necesariamente una buena herramienta para promover el desarrollo local: i) drenaje de los beneficios del proyecto a otras comunidades más alejadas, ii) mejora de infraestructuras que puedan animar las importaciones a través del puerto que mejoran su competitividad con respecto a productores locales, iii) potencial mayor presión fiscal e impositiva necesaria para la realización del desarrollo portuario que afecta a ciudadanos y empresas locales y iv) menor empleo local y contención de costes de mano de obra soportados por comunidades locales dado que la demanda agregada de mano de obra en una economía es determinada por efectos macroeconómicos, de tal manera que puertos compitiendo entre sí por una cuota de un mercado de tamaño dado puede derivar en la necesidad de implementar política de reducción de costes y adopción de medidas de mayor productividad. A estos efectos habría que añadir: v) la ocupación de la costa y la presión en precios del suelo externalidad soportada por las comunidades locales y, vi) los efectos que la congestión y operación cerca de umbrales de capacidad introduce en las zonas de influencia del puerto (Musso et al., 2011).

Acosta-Seró et al. (2009) sintetizan otras debilidades de la metodología de TIO que engloban en varios grupos genéricos. Los primeros de carácter técnico son el ya citado carácter estático de los estudios que supone a fin de cuentas que los inputs son proporcionales a los outputs (supuesto de linealidad) y que los precios relativos son fijos; la no incorporación de restricciones en la demanda o economías de escala, efectos sinérgicos o cambios tecnológicos que afecten a las relaciones intersectoriales. Otros más específicos del caso portuario se basan en la inexistencia de una definición comúnmente aceptada de industria portuaria, la adopción de procedimientos alternativos que dificulta la comparación de resultados y las debilidades cuando estos estudios son utilizados para estimar cambios en el volumen de servicios prestados en el puerto (consideración media versus marginal). Como autores en los que se apoyan para citar estas debilidades se encuentran los ya citados Waters (1977), Chang (1978), Davis (1983) y otros más recientes como Verbeke y Debisschop (1996) o Weisbrod y Weisbrod (1997).

En una línea parecida, Danielis y Gregori (2012) ponen de manifiesto que es importante conocer las diferencias metodológicas que existen entre unos y otros estudios para evitar errores de interpretación o establecer comparaciones equivocadas. Estos mismos autores han sido incluso críticos con el empleo de estos estudios con la finalidad arriba señalada indicando cómo es fácil exagerar el impacto de los puertos en su zona de influencia, poniendo de manifiesto que con el trascurso del tiempo y en algunas ocasiones los puertos ni generan todo el empleo que indican estudios anteriores, ni sirven a las industrias situadas en el entorno inmediato del puerto sino a otras más alejadas de la zona donde están establecidos y, por tanto, no reportando los beneficios locales que se pretenden poner de manifiesto para conseguir el apoyo de las comunidades. Utilizan para ello un análisis de los puertos de Plymouth (UK).

\section{A idénticas conclusiones habían llegado Gripaios y Gripaios (1995) y Gripaios (1999).}

La posible sobreestimación de los efectos económicos de un puerto ya había sido enfatizada por Davis (1983) y Verbeke y Debisschop (1996). Para los primeros, la falta de datos llevaba a una subjetiva valoración del grado de dependencia del puerto de las actividades desarrolladas en el mismo (fundamentalmente a lo que efectos indirectos se refiere). Para los segundos, incluso en EIEP bien formulados, no se tienen en cuenta ciertas externalidades y no permiten la consideración de precios sociales o en sombra con la que evaluar ciertos inputs y outputs (los estudios usualmente consideran el valor añadido a precios de mercado).

Rúa-Ferreño et al. (2014) defienden el uso de la metodología TIO en puertos, aplicando a un ejercicio práctico en los cuatro puertos gestionados por la Autoridad Portuaria de Baleares 
(Islas Baleares, España). Comienzan el estudio poniendo de manifiesto las ventajas del método sin ocultar más adelante sus principales defectos. Dentro de las ventajas indican que siendo un método basado en equilibrio general permite observar el conjunto de relaciones intersectoriales mientras que a su vez permite diferenciar tres niveles de impacto (directo, indirecto e inducido) y es quizá uno de los enfoques más neutrales puesto que se realizan pocos juicios de valor y los que se hacen, se explicitan. En cuanto a los inconvenientes destaca los clásicos en relación a la existencia de recursos ociosos, los que tienen que ver con la rigidez de precios y relaciones lineales entre variables y, por último, la falta de actualización de las tablas que sirven de base e indisponibilidad de datos.

Otros autores como Villaverde-Castro y Maza-Fernández (2015) justifican el desarrollo de EIEP como un procedimiento alternativo a otros métodos para el análisis de la competencia y competitividad de un puerto frente a otros. Estos otros métodos serían aquellos basados en el estudio comparado de productividad y eficiencia, los estudios de matrices de competitividad interportuaria o los métodos basados en los estudios de desviaciones de tráfico, hinterland y foreland de los puertos en competencia.

Para estos autores un puerto con mayor impacto económico en su hinterland es más competitivo pues, a igualdad de otras condiciones, presenta ventajas tanto del lado de la demanda (al tener mayor clientes, proveedores y usuarios es menos sensible a descensos coyunturales de ésta), como de la oferta (un puerto de mayor impacto en la economía tiene más facilidad para lograr el apoyo social para realizar sus ampliaciones evitando que los ciudadanos se opongan por razones ecológicas o ambientales) como de la inversión (un puerto con mayor impacto presenta un mayor atractivo para las inversiones privadas).

\section{Estudios Coste / Beneficio y su comparación con los EIE utilizando metodología TIO.}

La distribución de los beneficios que una inversión portuaria produce es un tema tratado desde hace muchos años (Laing, 1977) que es abordado desde diversas metodológicas al margen de los EIE. Entre otros destacan los Análisis Coste - Beneficio (referenciados en adelante como CBA por su extendido uso en literatura científica derivado de su acrónimo en inglés), los Análisis Coste - Efectividad (referido como CEA por idénticos motivos) o los más completos análisis multicriterio.

Mientras los CBA ponen su acento en poner valor monetario a la medida del efecto que un determinado proyecto de inversión introduce, los CEA focalizan su análisis en el diferencial de costes entre dos situaciones (con o sin proyecto) en relación a sus resultados (efectos).

Los análisis CBA son la técnica de evaluación de proyectos de inversión más extendida, probablemente porque presentan los resultados de una manera comprensible cuantificando monetariamente cómo un proyecto contribuye al bienestar económico y social.

Como en cualquier procedimiento de evaluación, para entender debidamente su bondad es necesario conocer las hipótesis de partida (supuestos), las limitaciones metodológicas inherentes (simplificaciones) y los objetivos que trata de cubrir (retos y alcance del CBA) aspectos que deben quedar explicitados a la hora de presentarlo.

Los CBA tratan de evaluar las inversiones mediante el cálculo y comparación de costes y beneficios en dos escenarios (con y sin proyecto) a lo largo de un periodo de tiempo y calculando el Valor Actual Neto (VAN) del mismo. 
Usualmente se abordan sobre la base de excedentes netos, realizando hipótesis de cómo evolucionaría la economía durante el periodo de evaluación hasta el año horizonte si el proyecto no se realizase y comparando este escenario con lo que pasaría si el proyecto se ejecutase.

En ocasiones se presentan los resultados en términos del ratio Beneficio (B) / Coste (C), entendido como el cociente entre los ahorros netos para el consumidor y los costes netos de capital y de operación y mantenimiento (Collier \& ve Ledbetter, 1988), calculados mediante la siguiente expresión:

$$
B / C=\frac{\text { Ahorros Netos para el consumidor }}{\text { Costes Netos de Capital }+ \text { Costes Netos de Operación y mamtenimiento }}
$$

Los CBA están ampliamente extendidos para la evaluación de proyectos de inversión en materia de infraestructuras y, dentro de éstos han encontrado también un amplio desarrollo en el caso portuario (CBAP).

Los primeros CBAP trataban de determinar los beneficios generados por un proyecto de inversión a través del cálculo de los ahorros en coste que para el puerto y los armadores suponía la reducción del tiempo de paso de mercancía por el puerto y la cuestión se centraba en determinar si esos beneficios alcanzaban el consumidor (reducción en fletes y costes portuarios repercutidos en el coste final del producto) o los capturaban las navieras $\mathrm{u}$ otros agentes portuarios (en un mercado imperfecto en ausencia de competencia (Wanhill, 1978).

En el caso de los CBAP es preciso recibir relevantes inputs de naturaleza técnica y que tienen que ver con la forma de evaluación de las capacidades (oferta portuaria) y cómo evolucionarán los tráficos (demanda portuaria). Con estos datos se debe conformar una estructura económica en el escenario sin proyecto (afectada, o no, por restricciones de capacidad a partir de determinado año e idealmente afectada por una estructura de costes que capture los fenómenos de saturación) y otra con proyecto (igualmente afectada, o no, por dichas restricciones pero como norma general en un año posterior a la situación sin proyecto toda vez que el objeto de la inversión suele ser un aumento de las capacidades portuarias con la construcción de nuevas obras de infraestructura -diques, muelles, explanadas de almacenamiento o aumentos de calado- o superestructura portuaria -grúas, almacenes...- o eliminación de cuellos de botella que la insuficiencia de infraestructura o superestructura introduce).

El cálculo suele abordarse mediante la estimación del excedente para el consumidor que se genera por la propia inversión y por los propios agentes (Autoridad Portuaria y usuarios y prestatarios de servicios en el puerto).

Para ello se suele discretizar el modelo calculando los costes y beneficios que generan tres categorías de tráfico: los que se mueven en el puerto en el año cero de la evaluación (tráficos existentes), los que se mueven en otros puertos, pero son susceptibles de ser captados en las nuevas instalaciones (tráficos desviados) y los que no existen a la fecha y se generarán a lo largo de la vida del proyecto (tráficos generados). No cabe duda que en una adecuada y creíble determinación de estos niveles de demanda radicará en gran medida la bondad de los resultados.

La forma de contabilizar los excedentes pasa por estimar los diferenciales de coste por disminución del tiempo de estancia de la mercancía en el puerto (causado por ejemplo por la mejora de la eficiencia que introduce las nuevas instalaciones) las menores distancias de transporte terrestre (típico efecto en caso de tráficos desviados asumiendo que tal desvío se producirá) o los beneficios para el consumidor por la aparición de nuevos tráficos. En la Tabla 5 presentamos a título de ejemplo como de Rus y Betancort (2012) abordan dichos cálculos para el caso concreto de la ampliación del Puerto de Sagunto (Valencia, España). 
Tabla 5. Fórmulas de evaluación de excedente de consumidor.

\begin{tabular}{|l|l|}
\hline Categoría Tráfico & Evaluación del Excedente del Consumidor \\
\hline Tráficos Existentes & $\Delta E C=\sum_{i}\left(g_{i t}^{0}-g_{i t}^{1}\right) q_{i t}^{0}=V_{t}\left(\tau_{i t}^{0}-\tau_{i t}^{1}\right) q_{i t}^{0}$ \\
\hline Tráficos Desviados & $\Delta E C=\sum_{i}\left(g_{i t}^{0}-g_{i t}^{1}\right)\left(q_{i t}^{0}-q_{i t}^{1}\right)=V_{t}\left(\tau_{i t}^{2}-\tau_{i t}^{1}\right)\left(q_{i t}^{0}-q_{i t}^{1}\right)$ \\
\hline Tráficos Generados & $\Delta E C=\frac{1}{2} \sum_{i}\left(g_{i t}^{0}-g_{i t}^{1}\right)\left(q_{i t}^{0}-q_{i t}^{1}\right)=\frac{1}{2} V_{t}\left(\tau_{i t}^{0}-\tau_{i t}^{1}\right)\left(q_{i t}^{0}-q_{i t}^{1}\right)$ \\
\hline
\end{tabular}

$\triangle E C$ es la variación del excedente del consumidor

$g_{i t}^{0} \quad$ es el precio generalizado sin proyecto para cada tráfico "i”"

$g_{i t}^{1} \quad$ es el precio generalizado con proyecto para cada tráfico "i”

$q_{i t}^{0} \quad$ es el tráfico sin proyecto (con o sin restricciones) para cada tráfico "i"

$q_{i t}^{1} \quad$ es el tráfico con proyecto (con o sin restricciones) para cada tráfico "i”"

$\mathrm{V}_{\mathrm{t}} \quad$ es el valor del tiempo para una tonelada de mercancía

$\tau_{i t}^{0} \quad$ es el tiempo de estancia para cada tipo de tráfico “i” en situación sin proyecto y en el puerto en el que se está realizando la evaluación

$\tau_{i t}^{1} \quad$ es el tiempo de estancia para cada tipo de tráfico “i” en situación con proyecto y en el puerto en el que se está realizando la evaluación

$\tau_{i t}^{2} \quad$ es el tiempo de estancia para cada tipo de tráfico "i” en situación sin proyecto en el puerto desde el que se produce el desvío

Fuente: Elaboración propia basada en de Rus \& Betancort (2012).

Varias son las simplificaciones más habituales en los CBA portuarios: i) no cuantificación de los impactos ambientales y las externalidades negativas que éstas ocasionan, ii) no consideración de los fenómenos de congestión y el coste que esto introduce en el sistema, iii) no consideración de las externalidades positivas que introducen los denominados Wider Economic Benefits (WEB) de difícil cuantificación y que tienen que ver con que los precios de bienes y servicios difieren del coste para la sociedad en su conjunto (por efectos tales como aglomeración, cambios de producción en mercados imperfectos o efectos en los ingresos fiscales desde el mercado laboral) (UK Department of Transport, 2018), iv) no consideración de los efectos indirectos bajo la premisa de que no existen distorsiones significativas en estos mercados secundarios (de Rus \& Betancort, 2012) o v) consideración durante toda el periodo de evolución del proyecto de los valores medios en cuanto al coste del paso de la mercancía por puerto o al coste del tiempo de la misma (ambos medidos en €/tonelada) sin considerar el cambio que la estructura de tráficos puede introducir en ambos parámetros o las necesidades de ajuste de fletes, tasas o tarifas portuarias ante la reacción comercial de la competencia del puerto, otros armadores, u operadores logísticos.

Mientras ha existido cierta tendencia a minusvalorar los EIE frente a los CBA, muchos son los autores que desde hace años los ven como herramientas complementarias para valorar el retorno esperado de infraestructuras del transporte desde una perspectiva multicriterio (Waters, 1977; Grosdidier De Matons, 1986; Hawkins, 1991). 
En línea con estos autores Verbeke y Debisschop (1996) detectan diez principales fuentes de crítica a los EIE pero argumentan como las mismas pueden ser aplicadas a los CBA u otras herramientas de evaluación (Tabla 6).

Tabla 6. Principales deficiencias de los EIE y su comparación con los CBA y otras herramientas de evaluación.

\begin{tabular}{|c|c|}
\hline Elemento de crítica a los EIE & Comparación entre EIE y CBA \\
\hline $\begin{array}{l}\text { Los EIE no consideran cambios marginales en la } \\
\text { estructura de precios de inputs outputs como sí lo } \\
\text { hacen los CBA. }\end{array}$ & $\begin{array}{l}\text { La realización de un EIE en el que se comparen } \\
\text { situaciones con y sin proyecto permite realizar un } \\
\text { análisis marginal. }\end{array}$ \\
\hline $\begin{array}{l}\text { Ciertos elementos de los EIE como salarios, } \\
\text { depreciación o costes financieros son } \\
\text { computados como "beneficios" en los EIE, } \\
\text { mientas que son computados como "coste" en los } \\
\text { CBA. De esta manera puede ocurrir que los } \\
\text { proyectos con mayores impactos socio- } \\
\text { económicos sean los de mayor coste y no resulten } \\
\text { en una asignación razonable de unos recursos } \\
\text { limitados. }\end{array}$ & $\begin{array}{l}\text { Algunas componentes de valor añadido como los } \\
\text { salarios pagados en época de construcción de la } \\
\text { infraestructura son considerados como "beneficios" } \\
\text { en los CBA y no capturados por los EIE, siendo más } \\
\text { correcto este enfoque si se trata de evaluar los } \\
\text { beneficios "sostenibles" (perdurables) y no } \\
\text { distorsiones temporales. }\end{array}$ \\
\hline $\begin{array}{l}\text { Los criterios de selección (o rechazo) de un } \\
\text { proyecto de inversión utilizando EIE sólo se } \\
\text { basan en el impacto del proyecto sobre } \\
\text { determinadas variables (impacto en el valor } \\
\text { añadido y empleo usualmente), ignorando otros. }\end{array}$ & $\begin{array}{l}\text { Los CBA también utilizan como criterio que el VAN } \\
\text { del proyecto sometido a evaluación sea mayor que } \\
\text { cero (asumiendo que no existen restricciones } \\
\text { presupuestarias o elementos de priorización de los } \\
\text { recursos públicos, la selección de proyectos se da } \\
\text { bajo este único criterio). Es necesario realizar un } \\
\text { análisis multicriterio para la selección de proyectos } \\
\text { de inversión. }\end{array}$ \\
\hline $\begin{array}{l}\text { Las políticas públicas pueden estimular la } \\
\text { economía (valor añadido o empleo) con otras } \\
\text { medidas macroeconómicas (reducción de } \\
\text { impuestos o políticas de subsidios a } \\
\text { determinados sectores) y no sólo a través de la } \\
\text { construcción de infraestructuras del transporte y } \\
\text { los EIE no abordan otras medidas alternativas y } \\
\text { aspectos relacionados con costes de oportunidad. }\end{array}$ & $\begin{array}{l}\text { Este elemento puede resultar válido desde la } \\
\text { perspectiva de la economía del bienestar, pero: i) } \\
\text { ignora las dificultades y constricciones } \\
\text { presupuestarias inherentes a todo proceso de } \\
\text { reducción de impuestos o políticas de subsidios y ii) } \\
\text { no es claro que cambios relativamente modestos en } \\
\text { fiscalidad puedan producir cambios significativos en } \\
\text { la actividad económica. En muchas ocasiones las } \\
\text { inversiones en materia de infraestructura introducen } \\
\text { un efecto psicológico que sirve de catalizador de un } \\
\text { incremento de la actividad económica. } \\
\text { Adicionalmente este es un elemento que aplica a } \\
\text { otras metodologías si no se evalúan los costes - } \\
\text { beneficios de estas otras medidas. }\end{array}$ \\
\hline $\begin{array}{l}\text { Los EIE se utilizan más como una herramienta de } \\
\text { relaciones públicas que como una herramienta } \\
\text { seria de evaluación de proyectos. }\end{array}$ & $\begin{array}{l}\text { Los CBA (y otros tipos de análisis) no están exentos } \\
\text { de este riesgo si: i) se ocultan las externalidades } \\
\text { negativas que producen, ii) si se realizan } \\
\text { simplificaciones metodológicas con intención de } \\
\text { alterar el resultado (por ejemplo, maximizando } \\
\text { beneficios y disminuyendo costes) o si, iii) se toman } \\
\text { hipótesis en esa misma dirección (Verbeke, 1988). }\end{array}$ \\
\hline $\begin{array}{l}\text { La asunción de que una inversión en } \\
\text { infraestructura y el impacto económico que esta } \\
\text { produce se traduce en un cambio en la visión }\end{array}$ & $\begin{array}{l}\text { Este mismo "acto de fe" se requiere en los CBA } \\
\text { cuando por ejemplo se estima que se producirá un } \\
\text { desvío de tráfico y cambio de puerto por parte de una }\end{array}$ \\
\hline
\end{tabular}




\begin{tabular}{|c|c|}
\hline $\begin{array}{l}\text { estratégica de las empresas (sus directivos) o } \\
\text { emprendedores provocando un efecto } \\
\text { multiplicador y cambio de las relaciones entre } \\
\text { sectores (nuevas tablas TIO en situación con } \\
\text { proyecto) es más un "acto de fe" que un hecho } \\
\text { analítico demostrable. }\end{array}$ & $\begin{array}{l}\text { mercancía al construir una nueva infraestructura por } \\
\text { el simple hecho de que se produzca una reducción } \\
\text { del coste del transporte (obviando otros elementos } \\
\text { que conforman la toma de decisión). }\end{array}$ \\
\hline $\begin{array}{l}\text { Los efectos multiplicadores y la evaluación de } \\
\text { los efectos indirectos son usualmente } \\
\text { sobreestimados en los EIE. }\end{array}$ & $\begin{array}{l}\text { Una correcta confección de tablas TIO en situación } \\
\text { con proyecto y la adopción de hipótesis razonables } \\
\text { de cómo responderá la economía tras la ejecución } \\
\text { del proyecto es la garantía para solventar esta } \\
\text { carencia. Muchos CBA no hacen ni siquiera el } \\
\text { intento de evaluar los efectos indirectos que } \\
\text { consideran despreciables. }\end{array}$ \\
\hline $\begin{array}{l}\text { Es muy difícil disociar los impactos que produce } \\
\text { una determinada infraestructura (por ejemplo el } \\
\text { puerto) de los que producen otras infraestructuras } \\
\text { de acompañamiento (por ejemplo, las carreteras } \\
\text { o ferrocarriles que hacen falta para su } \\
\text { funcionamiento) computándose todo el impacto } \\
\text { positivo al primero. }\end{array}$ & $\begin{array}{l}\text { Este problema se reproduce en muchos CBA cuando } \\
\text { no se consideran los costes de estas infraestructuras } \\
\text { de acompañamiento, pero sí se computa todo el } \\
\text { beneficio a la nueva infraestructura. En ambos casos } \\
\text { CBA y EIE deben o bien considerarse todos los } \\
\text { elementos o bien dejar claro cuáles no se tienen en } \\
\text { cuenta y en qué medida ayudan a sobre-estimar los } \\
\text { efectos positivos. }\end{array}$ \\
\hline $\begin{array}{l}\text { Los EIE no tienen en consideración efectos } \\
\text { externos y no permiten tener en consideración } \\
\text { precios en la sombra para la valoración de inputs } \\
\mathrm{u} \text { outputs específicos (proporcionando una } \\
\text { realidad distorsionada desde la perspectiva de la } \\
\text { eficiencia de la economía). }\end{array}$ & $\begin{array}{l}\text { Los CBA u otras herramientas de evaluación } \\
\text { adolecen de similares deficiencias al no considerar } \\
\text { todas las implicaciones que para los agentes (en el } \\
\text { caso portuario la Autoridad Portuaria, los usuarios o } \\
\text { los prestatarios de servicios) tienen efectos no } \\
\text { cuantificables desde la perspectiva del coste o } \\
\text { beneficio (ciertas contribuciones marginales de } \\
\text { proyectos de inversión en la economía de la zona } \\
\text { como los ya mencionados WEB). }\end{array}$ \\
\hline
\end{tabular}

Fuente: Elaboración propia basada en Verbeke \& Debisschop (1996).

Estos últimos autores presentan una posible división en cuatro cuadrantes de las metodologías de evaluación de proyectos basándose en la naturaleza y alcance de los impactos evaluados (Figura 1) y sin proporcionar una guía de en qué cuadrante es más adecuado realizar una u otra metodología si indican como según nos vamos moviendo del primer cuadrante al cuarto cuadrante los estudios son más completos a costa de un mayor nivel de incertidumbre y, a veces, un menor rigor de los estudios. Si clasifican estos autores los proyectos en tres categorías según interese la evaluación del valor añadido y empleo que generan pues ésta es la principal razón por la que se promueven (para lo que se recomienda como técnica de evaluación los EIE), aquellos donde éstas no son las únicas razones para promover el proyecto (en cuyo caso deberían evaluarse mediante sistemas multicriterio que capturase el conjunto de variables impulsoras del mismo) y los que se promueven buscando otros impactos tales como reducción de la congestión u otras externalidades y efectos externos y no necesariamente el incremento del valor añadido o el empleo (proyectos en los que el análisis CBA parecen más indicados si son capaces de cuantificar dichos efectos). 
Figura 1. Clasificación de herramienta de evaluación económica por la naturaleza y alcance de los impactos analizados.

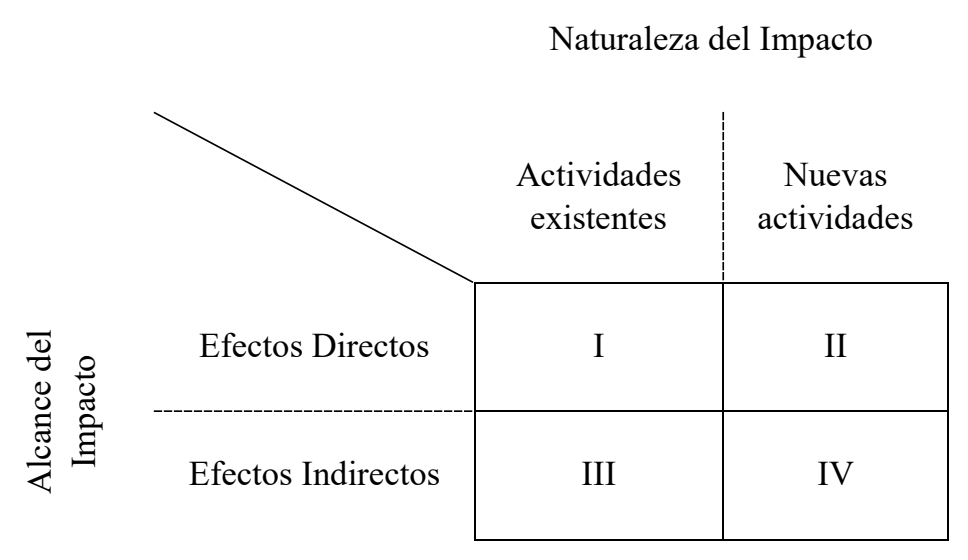

Fuente: Rediseñado de Verbeke \& Debisschop (1996).

En nuestra experiencia, los EIE suelen enmarcarse más en el cuadrante I-III analizando los impactos directos e indirectos de actividades existentes sobre las que se conocen las relaciones inter-sectoriales y se cuenta con el apoyo de tablas TIO (si bien hay algunos estudios que también lo hacen en el II-IV al considerar el efecto de las inversiones y nuevas líneas de actividad de las que sirven de catalizador); mientras que los CBA se mueven típicamente en el cuadrante I-II (evaluación de efectos directos de las actividades existentes y las que generará la nueva infraestructura), si bien hay algunos estudios en los que se consideran también elementos del cuadrante II-IV (ampliando el estudio a los efectos indirectos).

\section{Resultados obtenidos, conclusiones y recomendaciones para próximas líneas de investigación.}

En el año 2014, la Organización para la Cooperación Económica y Desarrollo, publicó un monográfico sobre competitividad de ciudades portuarias (OECD, 2014) en las que se sintetizaba un conjunto de estudios sobre regiones portuarias concretas realizadas en la primera mitad de la década del 2010 sobre un conjunto de puertos en Europa, Asia, América del Sur y África (Merk et al., 2011; Merk \& Comtois, 2012; Merk \& Hesse, 2012; Merk et al., 2012; Merk, 2013a, 2013b; Merk \& Bagis, 2013; Merk \& Li, 2013; Hong et al., 2013; van den Bossche et al., 2013).

En esta serie se incluían estudios utilizando metodología TIO y se ofrecían interesantes conclusiones en relación al tamaño del puerto / región y su capacidad de generar empleo y VAB (medidos a efectos de competitividad en unidades per cápita).

Al tiempo se incidía en el que al tradicional impacto reportado mediante esta metodología (usualmente en términos de empleo y VAB incluyendo efectos directos, indirectos e inducidos) había que añadir una cuarta categoría de impacto denominado catalítico y que son aquellos generados por el puerto como elemento facilitador de incrementos de productividad y la capacidad de atraer de nuevos negocios a la región por ello (Ferrari et al., 2012). Se trata como hemos visto de la consideración de parte de los WEB en los EIEP.

En este estudio se demostraba que cuanto más grande era un puerto, mayor era su capacidad para generar valor añadido y para ello se apoyaban empíricamente en un estudio de gran escala que analizaba 150 EIEP y en el que se concluía que, en promedio, cada tonelada movida por un puerto generaba 100 USD de valor añadido con $2 / 3$ partes de los puertos en el rango 50-250 USD/tonelada (impacto sin contar el impacto catalítico) pero con puertos de mayores dimensiones 
en la parta alta del rango. Para ello comparaban aquellos estudios con metodología similar y se limitaban a incluir impactos directos e indirectos.

En este mismo estudio se deduce cómo la capacidad de generar valor también depende del tipo de mercancía manipulada, y sugiriendo, teniendo en cuenta una muestra de puertos en Estados Unidos, que la carga general y contenedorizada puede generar 10 veces más valor que la de graneles. Así, en el caso de tráfico de automóviles el valor añadido alcanzaba de media 220 $\mathrm{USD} / \mathrm{tn}$, bajando a $90 \mathrm{USD} / \mathrm{tn}$ en el caso de contendores, $60 \mathrm{USD} / \mathrm{tn}$ para tráficos siderúrgicos, $45 \mathrm{USD} / \mathrm{tn}$ para productos petrolíferos y $20 \mathrm{USD} / \mathrm{tn}$ para el grano.

En el mismo estudio se indica un rango de multiplicador de Leontieff de la actividad portuaria que variaba entre 2,47 (Le Havre/Rouen) y 1,13 (Rotterdam) y que incluía otros puertos europeos como Marsella $(2,01)$, Mersin $(1,79)$, Hamburgo $(1,71)$ y Amberes $(1,18)$.

Rodríguez Dapena (2005) realiza una revisión de los EIEP realizados en el caso del Sistema Portuario Español (SPE) que es más tarde completada y actualizada por de la Peña et al. (2018b) quienes utilizan coeficientes deflactores para armonizar los datos al año 2016. De estos estudios se concluye que en promedio el sector portuario del SPE tiene un impacto de $27 € /$ Tonelada, una capacidad de generación de empleo de 418 empleos por millón de tonelada y una productividad (medida en términos de VAB por empleo) de $75.824 € /$ empleo. Si se amplía el foco teniendo en consideración la economía dependiente del puerto los resultados suben a $100 € /$ Tonelada, 1.812 empleos/millón de tonelada y $59.710 € /$ empleo (Tabla 7). Dichos datos presentan, sin embargo, una amplia dispersión que tiene que ver con el tamaño y la estructura de tráficos de dichos puertos pudiendo consultarse los datos individualizados por puerto en función de los EIEA analizados en el Anexo 1.

Tabla 7. Impacto Económico de los Puertos Españoles.

\begin{tabular}{|c|c|c|c|c|c|c|}
\hline & \multicolumn{3}{|c|}{ Sector Portuario } & \multicolumn{3}{|c|}{ Economía Dependiente } \\
\hline & $\begin{array}{c}\mathrm{VAB} / \mathrm{tn} \\
(€ / \mathrm{Tn})\end{array}$ & $\begin{array}{c}\text { Empleo/ } \\
10^{6} \mathrm{tn}\end{array}$ & $\begin{array}{c}\text { Productividad } \\
\text { VAB/empleo } \\
\text { (€/empleo) }\end{array}$ & $\begin{array}{c}\text { VAB/tn } \\
(€ / T n)\end{array}$ & $\begin{array}{c}\text { Empleo/ } \\
10^{6} \mathrm{tn}\end{array}$ & $\begin{array}{c}\text { Productividad } \\
\text { VAB/empleo } \\
\text { (€/empleo) }\end{array}$ \\
\hline PROMEDIO & 27 & 418 & 75.824 & 100 & 1.812 & 59.170 \\
\hline MAXIMO & 71 & 1.246 & 232.263 & 576 & 7.591 & 111.231 \\
\hline MINIMO & 3 & 40 & 23.570 & 5 & 85 & 28.925 \\
\hline
\end{tabular}

Fuente: Elaboración propia basado en datos de Rodriguez Dapena (2005) y de la Peña et al. (2018b).

Como norma general, los EIEP analizados no tratan de evaluar las externalidades que, en materia ambiental, introducen la ejecución de inversiones portuarias (fase de construcción), las propias operaciones portuarias (fase de explotación) o, si procede, su posterior desmantelamiento (fase de abandono). Esto viene provocado por la dificultad y lo controvertido que en muchos casos supone evaluar de manera económica según qué impactos (por ejemplo, el paisajístico). La ausencia de consideración de externalidades ambientales en los EIEP es de hecho puesta de manifiesto por muchos autores (Davis, 1983; Verbeke \& Debisschop, 1996; Benaccio et al. 2000; Musso et al., 2011; Rus \& Betancort, 2012). Tan sólo algunos estudios como los anteriormente citados de la escuela china abordan el particular haciéndolo en cualquier caso de una manera muy parcial (fijándose en determinados aspectos de los impactos producidos como ocupación de suelo, consumos de agua o energía pero omitiendo otros muchos como aquellos que se originan como consecuencia de desplazamiento de otras actividades, impactos en otros sectores económicos, tráficos de congestión, impactos a flora o fauna, impactos paisajísticos, impactos relacionados con potenciales accidentes en zona portuaria...). El enfoque suele ser más bien el contrario, de tal manera que se incluye un análisis de los EIE del puerto en los Estudios de Impacto Ambiental (EIA) del mismo (usualmente asociados al desarrollo de nuevos proyectos de inversión) 
valorando éstos de una manera positiva y poniéndoles en contrapartida de los impactos negativos antes señalados. Como futura línea de investigación se propone analizar cuantos EIEP incorporan la componente ambiental y de qué manera cuantifican sus efectos

De la revisión realizada se han detectado las siguientes líneas de investigación en la que la comunidad científica podría profundizar:

1. Continuar con el establecimiento de una metodología estandarizada que permitiese una comparación de resultados con mayor rigor académico, haciendo hincapié en una normalización del concepto de industria dependiente del puerto.

2. Análisis detallado de la influencia del tamaño del puerto, ubicación geográfica y su estructura de tráficos a la hora de determinar el impacto del mismo.

3. Análisis dinámicos del impacto económico de los puertos mediante comparación de los resultados en un mismo puerto para distintos años de referencia.

4. Ampliación de estudios para determinación del impacto catalítico u otros WEB incluyendo una base metodológica común de referencia para el caso portuario.

5. Análisis de la relación entre los EIEP y los EIA analizando de qué manera los primeros tienen en consideración las externalidades negativas a la hora de evaluar el impacto económico de los puertos o cómo los segundos introducen el impacto económico como un elemento más a considerar en el balance de impactos dentro de la evaluación ambiental de estas infraestructuras. 
Tabla 8. Detalle del Impacto Económico de los Puertos Españoles por puerto.

\begin{tabular}{|c|c|c|c|c|c|c|c|c|}
\hline \multirow{2}{*}{ Puerto } & \multirow{2}{*}{ Año Estudio } & \multirow{2}{*}{$\begin{array}{c}\text { Coeficiente } \\
\text { Deflector }\end{array}$} & \multicolumn{3}{|c|}{ Sector Portuario } & \multicolumn{3}{|c|}{ Economía Dependiente } \\
\hline & & & $\mathrm{VAB}_{\mathrm{pm}} / \mathbf{t n}$ & Empleo/ $10^{6}$ tn & $\begin{array}{c}\text { Productividad } \\
\text { VAB/empleo }\end{array}$ & $\mathrm{VAB}_{\mathrm{pm}} / \mathbf{t n}$ & Empleo/ $10^{6}$ tn & $\begin{array}{l}\text { Productividad } \\
\text { VABpm/empleo }\end{array}$ \\
\hline Puertos de Galicia & 1992 & 1.620580 & 13.5 & 283 & 47,518 & 167.5 & 4,905 & 34,159 \\
\hline La Luz y las palmas & 1992 & 1.620580 & 36.8 & 598 & 61,580 & $\mathrm{n} / \mathrm{a}$ & $\mathrm{n} / \mathrm{a}$ & $\mathrm{n} / \mathrm{a}$ \\
\hline Santander & 1992 & 1.620580 & 38.7 & 945 & 40,953 & 342.2 & 7,591 & 45,086 \\
\hline Sta Cruz de Tenerife & 1992 & 1.620580 & 18.5 & 328 & 56,273 & $\mathrm{n} / \mathrm{a}$ & $\mathrm{n} / \mathrm{a}$ & $\mathrm{n} / \mathrm{a}$ \\
\hline Avilés & 1995 & 1.556436 & 43.7 & 606 & 72,057 & 142.8 & 1,919 & 74,408 \\
\hline Barcelona & 1995 & 1.556436 & 53.9 & 691 & 77,996 & $\mathrm{n} / \mathrm{a}$ & $\mathrm{n} / \mathrm{a}$ & $\mathrm{n} / \mathrm{a}$ \\
\hline Gijón & 1995 & 1.556436 & 22.0 & 212 & 103,650 & 58.2 & 825 & 70,535 \\
\hline Sevilla & 1995 & 1.556436 & 12.2 & 517 & 23,570 & 186.4 & 4,216 & 44,207 \\
\hline Tarragona & 1995 & 1.556436 & 11.3 & 114 & 99,814 & $\mathrm{n} / \mathrm{a}$ & $\mathrm{n} / \mathrm{a}$ & $\mathrm{n} / \mathrm{a}$ \\
\hline Bahía de Algeciras & 1996 & 1.515889 & 13.6 & 288 & 47,296 & 61.7 & 1,020 & 60,524 \\
\hline Ceuta & 1996 & 1.515889 & 12.2 & 513 & 23,865 & 51.9 & 1,287 & 40,343 \\
\hline Castellón & 1997 & 1.461949 & 19.9 & 293 & 67,804 & 61.7 & 749 & 82,449 \\
\hline Bahía de Cádiz & 1998 & 1.401597 & 22.4 & 410 & 54,630 & 221.4 & 3,898 & 56,800 \\
\hline Santander & 1998 & 1.401597 & 37.7 & 629 & 59,863 & 576 & 5,179 & 111,231 \\
\hline Bilbao & 1999 & 1.341436 & 19.4 & 362 & 53,701 & $\mathrm{n} / \mathrm{a}$ & $\mathrm{n} / \mathrm{a}$ & $\mathrm{n} / \mathrm{a}$ \\
\hline Avilés & 2000 & 1.274050 & 54.8 & 502 & 109,134 & 158.9 & 1,989 & 79,880 \\
\hline Barcelona & 2000 & 1.274050 & 42.7 & 533 & 80,084 & $\mathrm{n} / \mathrm{a}$ & $\mathrm{n} / \mathrm{a}$ & $\mathrm{n} / \mathrm{a}$ \\
\hline Cartagena & 2000 & 1.274050 & 4.7 & 88 & 53,468 & 25 & 507 & 49,247 \\
\hline Gijón & 2000 & 1.274050 & 9.8 & 140 & 70,144 & 79.6 & 1,038 & 76,687 \\
\hline Sevilla & 2000 & 1.274050 & $\mathrm{n} / \mathrm{a}$ & 886 & $\mathrm{n} / \mathrm{a}$ & $\mathrm{n} / \mathrm{a}$ & 2,744 & $\mathrm{n} / \mathrm{a}$ \\
\hline Marín & 2001 & 1.225036 & 33.4 & 1,130 & 29,574 & 69.4 & 2,400 & 28,925 \\
\hline Pasajes & 2001 & 1.225036 & 35.3 & 360 & 98,061 & 135.5 & 4,380 & 30,931 \\
\hline
\end{tabular}




\begin{tabular}{|c|c|c|c|c|c|c|c|c|}
\hline \multirow{2}{*}{ Puerto } & \multirow{2}{*}{ Año Estudio } & \multirow{2}{*}{$\begin{array}{c}\text { Coeficiente } \\
\text { Deflector }\end{array}$} & \multicolumn{3}{|c|}{ Sector Portuario } & \multicolumn{3}{|c|}{ Economía Dependiente } \\
\hline & & & $\mathrm{VAB}_{\mathrm{pm}} / \mathrm{tn}$ & Empleo/ $10^{6}$ tn & $\begin{array}{c}\text { Productividad } \\
\text { VAB/empleo }\end{array}$ & $\mathrm{VAB}_{\mathrm{pm}} / \mathbf{t n}$ & Empleo/ $10^{6}$ tn & $\begin{array}{l}\text { Productividad } \\
\text { VABpm/empleo }\end{array}$ \\
\hline Vigo & 2001 & 1.225036 & 44.1 & 959 & 45,958 & 135.3 & 4,377 & 30,900 \\
\hline Villagarcía de Arosa & 2001 & 1.225036 & 15.5 & 228 & 68,058 & 26.3 & 634 & 41,463 \\
\hline Algeciras & 2003 & 1.153961 & 3.2 & 40 & 77,970 & 4.8 & 85 & 55,653 \\
\hline Málaga & 2003 & 1.153961 & 41.9 & 769 & 54,513 & $\mathrm{n} / \mathrm{a}$ & $\mathrm{n} / \mathrm{a}$ & $\mathrm{n} / \mathrm{a}$ \\
\hline Castellón & 2004 & 1.118539 & $\mathrm{n} / \mathrm{a}$ & 49 & $\mathrm{n} / \mathrm{a}$ & 12.2 & 233 & 52,523 \\
\hline Las Palmas & 2005 & 1.078391 & 13.2 & 184 & 71,549 & 25 & 380 & 65,785 \\
\hline Santander & 2005 & 1.078391 & 38.6 & 380 & 101,575 & 99.6 & 1,711 & 58,223 \\
\hline Alicante & 2009 & 1.023119 & 7.3 & 126 & 58,279 & 15.9 & 307 & 51,820 \\
\hline Almería & 2009 & 1.023119 & 6.3 & 96 & 65,093 & 13.8 & 241 & 57,380 \\
\hline Ferrol & 2009 & 1.023119 & 19.1 & 357 & 53,420 & 34 & 803 & 42,340 \\
\hline Bilbao & 2010 & 1.022975 & $\mathrm{n} / \mathrm{a}$ & 147 & $\mathrm{n} / \mathrm{a}$ & $\mathrm{n} / \mathrm{a}$ & 361 & $\mathrm{n} / \mathrm{a}$ \\
\hline Cádiz & 2010 & 1.022975 & 71.0 & 1,090 & 65,137 & 136.4 & 2,565 & 53,165 \\
\hline Huelva & 2010 & 1.022975 & 31.4 & 135 & 232,263 & 62.1 & 685 & 90,742 \\
\hline Baleares & 2011 & 1.033296 & 64.7 & 1,246 & 51,908 & 116.7 & 2,280 & 51,160 \\
\hline Cartagena & 2011 & 1.033296 & 21.1 & 102 & 207,283 & 44.8 & 516 & 86,886 \\
\hline Algeciras & 2014 & 1.068191 & 12.8 & 102 & 124,765 & 20.9 & 264 & 79,136 \\
\hline Puertos de Andalucía & 2014 & 1.068191 & 23.6 & 188 & 125,727 & 43.5 & 616 & 70,686 \\
\hline Castellón & 2015 & 1.032745 & $\mathrm{n} / \mathrm{a}$ & 132 & $\mathrm{n} / \mathrm{a}$ & 20 & 367 & 54,472 \\
\hline Valencia & 2015 & 1.032745 & 25.7 & 362 & 70,953 & 34.7 & 528 & 65,688 \\
\hline \multicolumn{3}{|c|}{ PROMEDIO } & 26.9 & 418 & 75,824 & 99.5 & 1,812 & 59,170 \\
\hline \multicolumn{3}{|c|}{ MAXIMO } & 71.0 & 1,246 & 232,263 & 576 & 7,591 & 111,231 \\
\hline \multicolumn{3}{|c|}{ MINIMO } & 3.2 & 40 & 23,570 & 4.8 & 85 & 28,925 \\
\hline
\end{tabular}

Fuente: Elaboración propia basado en datos de Rodriguez Dapena (2005) y de la Peña et al. (2018b). 


\section{Referencias}

Acosta-Seró, M., Coronado-Guerreo, D., \& Cerbán-Jiménez, M.M. (2009). Evaluación del impacto económico del puerto de Tarifa: Situación actual y prospectiva tras la ampliación. Departamento de Economía General Universidad de Cádiz, Cádiz (España). Recuperado de http://noalmegapuerto.detarifa.net/files/EVAUALION-IMPACTO-ECONOMICO-PUERTOTARIFA.pdf.

Anderson, G.K. (1964). The port of Ensenada: A report on Economic Development. San Diego State College Foundation, Economics Research Center, Dept. of Economics, San Diego State College, California (USA).

Armenakis, A.A., Moore, H.J., \& Peden, G.T. (1970). Inland Port Facilities and Economic Growth. Mississippi State University, Bureau of Business and Economic Research, Mississippi (USA).

Arrow, K.J., \& Debreu, G. (1954). Existence of an Equilibrium for a Competitive Economy. Econometrica, 22(3), 265-290. Recuperado de https://web.stanford.edu/class/msande311/arrowdebreu.pdf.

Arthur D. Little, Inc. (1979). Port Economic Impact Kit. Washington: Dept. of Commerce, Maritime Administration, Office of Commercial Development, Office of Port and Intermodal Development, Washington DC (USA).

Beeman, O. Associattes \& Mannalytcis Inc. (1980). Oregon Port Study - 1980. Oregon Port Authorit, San Francisco (California, USA).

Behan, S. (1988). Economic Impact of Dublin Port on its Hinterland. Dublin Port, Dublín (Irlanda).

Bichou, K. (2007). Review of Port Performance Approaches and a Supply Chain Framework to Port Performance Benchmarking". En Mary Brooks \& Kevin Culliname (eds.) Devolution, Port Governance and Port Performance, (pp: 567-598). Stamford, (USA): JAI Press.

Booz-Allen \& Hamilton, Inc. (1982). The Economic Impact of the Port of Baltimore. The Maryland Port Authority, Baltimore (USA).

Bragg, D.M., \& Bradley J.R. (1972). The economic impact of a deep Water Terminal in Texas. Texas A\&M University, Texas Engineering Station, Texas (USA).

Bragg, D.M. (1974). A survey of the economic and environmental aspects of on Onshore Deepwater Port at Galveston, Texas, Part I. Potential Economics Effects. Industrial Research Division, Texas A\&M University, College Station, TAMUG SG-74-213, Texas (USA).

Braun, B.M. (1990). Measuring the influence of public authorities through economic impact analysis: The case of Port Canaveral. Policies Studies Journal, 18(4), 1032-1043. DOI: 10.1111/j.15410072.1990.tb00869.x.

Cao, Y. (2009). System Dynamics Analysis On Socio-Economic Impact of Coal Port Construction Projects. En Globe Thesis ( . Recuperado de http://www.globethesis.com/?t=2189360272486327.

Cardenete-Flores, A.M., \& López-Cabaco, R. (2016). Economic Impact of the Port of Algeciras's Rail Corridor in Andalusia: a Dynamic CGE approach. En International Conference on Regional Science: XLII Reunión de Estudios Regionales (pp.1-25), 16-18 de noviembre de 2016, Santiago de Compostela (España). 
Carew, J.P. (1975). Port of Stockton, Phase 2: Economic Impact of the Port of Stockton on the Stockton Economy 1973-1974. Port of Stockton, California (USA).

Chang, S. (1978). In Defense of Port Economic Impact Studies. Transportation Journal, 17(3), 79-85. Recuperado de https://www.jstor.org/stable/20712474.

Chen, W.-S., Chen. C.-Y., Chen, F.-C., \& Liu C.-C. (2011). The impact of the Taipei Port Container Terminal on the Northern Region of Taiwan: A computable general equilibrium model. Journal of Marine Science and Technology, 19(2): 120-126. Recuperado de http://jmst.ntou.edu.tw/marine/192/120-126.pdf.

Collier, A.A. \& ve Ledbetter, W.B. (1988). Engineering Economy and Cost Analysis. Harper \& Row Publications, New York (USA).

Condor, W. \& Tynerson, R. (1972). Survey of Oregon Ports, 1972. Economic Impact Section. Oregon Department of Transportation, Ports Division, Oregón (USA).

Conn, R.L., Flewellen, W.G., \& Peden, G.T. (1966). An assessment of Major Benefits to Mississippi from Waterborne Commerce. State College, Mississippi State University, Bureau of Business and Economic Research. Mississippi (USA).

Conway, D. \& Associates (1989). 1987 Economic Impact Study. Technical Report. Port of Seattle, Seattle (USA).

Costacurta de Sá Porto, P. (2010). What Role of the Port in Brazilian States Exports: a Gravity Model Approach. En XXXIV Encontro da ANPAD (pp: 1-13), 25-29 de septiembre de 2010 Rio de Janiero (Brasil). Recuperado de http://www.anpad.org.br/admin/pdf/gol944.pdf.

Coto-Millán, P., Mateo-Mantecón, I. \& Villaverde-Castro, J. (2010b). A methodological discussion on port economic impact studies and their possible applications to policy design. En Coto-Millán, P., Pesquera, M.A. \& Castanedo, J. (Eds.). Essays on Port Economics (pp: 151-160). Springer-Verlag Berlin Heidelberg.

Crompton, J.L. (2006). Economic Impact Studies: Instruments for Political Shenanigans?. Journal of Travel Research, 45(1), 67-82. DOI: 10.1177/0047287506288870.

Cui, J.X. (2007). A Study of Yangtze River Delta Port Group Logistics System based on system dynamics developing In Correspond. En Globe Thesis (C) Recuperado de http://www.globethesis.com/?t=2189360212455725.

Dai, X.J. (2012). The Port Development Research Based On Hinterland's Economy And Competition And Cooperation Among Port Group. En Globe Thesis (C) Recuperado de http://www.globethesis.com/?t=2249330362968023.

Danielis, R., \& Gregori, T. (2012). An input-output based methodology to estimate the economic role of a port: the case of the port system of the Friuli Venezia Giulia Region, Italy. En Working Papers of SIET 2012 (Italian Association of Transport Economics), 28-30 de junio de 2012, Bari (Italia).

Danielis, R., \& Gregori, T. (2013). An input-output based methodology to estimate the economic role of a port: the case of the port system of the Friuli Venezia Giulia Region, Italy. Maritime Economics \& Logistics, 15(2), 222-255. DOI: 10.1057/mel.2013.1.

Davis, H.C. (1983). Regional Port Impact Studies: a critique and suggested methodology. Transportation Journal, 23(2):61-71. Recuperado de https://www.jstor.org/stable/20712762. 
de la Peña, I. (2018a). Historia, Evolución y Perspectivas de Futuro en la utilización de técnicas de simulación en la Gestión Portuaria: Aplicaciones en el análisis de operaciones, estrategia y planificación portuaria. Tesis Doctoral. Universidad de A Coruña (A Coruña, España). Recuperado de https://ruc.udc.es/dspace/handle/2183/20927.

de la Peña, I., Freire, M.J., \& López, B. (2018b). Impacto económico del puerto de Ferrol - San Cibrao: Armonización de resultados al año de referencia 2016 y comparación con los puertos estatales. Revista Galega de Economía, 27(2), 33-48. Recuperado de https://ideas.repec.org/a/sdo/regaec/v27y2018i2_3.html.

De Salvo, J. \& Fuller, D. (1988). The economic impact of the Port of Tampa. Center for Economic and Management Research, Tampa (Florida, USA).

De Salvo, J., \& Fuller, D. (1995). The role of Price Elasticities of Demand in the Economic of a Port. The Review of Regional Studies, 25(1), 13-35. Recuperado de https://ideas.repec.org/a/rre/publsh/v25y1995i1p13-35.html.

De Salvo, J.S. (1994). Measuring the Direct Impacts of a Port. Transportation Journal, 33(4), 33-42. Recuperado de https://www.jstor.org/stable/20713212.

De Rús, G., \& Betancort, O. (2012). Análisis Coste - Beneficio de Proyectos Portuarios. Papeles de Economía Española, 131, 92-105. Recuperado de https://accedacris.ulpgc.es/bitstream/10553/70297/1/Analisis_coste_beneficio.pdf.

Doi, M., Tiwari, P., \& Ito, H. (2002). A computable General Equilibrium Analysis of Efficiency Improvements at Japanese Ports. Review of Urban and Regional Development Studies, 13(3), 187206. DOI: 10.1111/1467-940X.00040.

Dunphy, L.M., \& Chang, S. (1973). Economic Analysis of the Port of Mobile with special reference to its economic impact upon the Alabama Economy 1973. Department of Economics, University of South Alabama, Mobile (Alabama, USA).

Dwarakisha, G.S., \& Salima, A.K. (2015). Review on the Role of Ports in the Development of a Nation. Aquatic Procedia, 4 (2015), 295-301. DOI: 10.1016/j.aqpro.2015.02.040.

Economics Research Associates (1976). Community Economic Impact of the Marine Terminals of Port of Portland. Port of Portland, Los Angeles, California (USA).

Erbuth, H. (1966). Der Betrag eines Hafens zur Wetschiepfung seiner Hafensadt garfestellt am Beispeil der Haefen Hamm und Neuss, En Dietmar Kraftt (editor) Der Eingluss eines Hafens aud die Wirtschaftsstruktur und die Wirtschftskraft seiner Hafenstadt, Goettingen (Alemania).

Ferrari, C., Merk, O., Bottasso, A., Conti, M. \& Tei, A. (2012). Ports and Regional Development: a European Perspective. OECD Regional Development Working Paper 2012/07. París. Francia. DOI: $10.1787 / 20737009$.

Francis, N.J. (1970). The urban growth and transportation implications in port development. A case of study, Vancouver, British Columbia. Tesis Doctoral no publicada. University of British Columbia, Vancouver (Canadá).

Fu, M.M. (2011a). Port and Economy Relationship Analysis by System Dynamics. En Proceedings of the International Conference on Transportation Engineering (ICTE), pp. 162-16). Julio 2011, Chengdu (China). 
Fu, M.M. (2011b). A Study On Port-Regional Economy System Based On System Dynamics. En Globe Thesis (C). Recuperado de http://www.globethesis.com/?t=1119360308969767.

Gao, S. (2012). Research on the regional development of Dalian Port based on system dynamics. En Globe Thesis ( ). Recuperado de http://www.globethesis.com/?t=2189330335455484.

Goss, R.O. (1990). Economic Policies and Seaport: The Economic Functions of Seaports. Maritime Policy and Management, 17(3), 207-219. DOI: 10.1080/03088839000000028.

Gripaios, P. (1999). Ports and their influence on local economies: a UK perspective. The Dock \& Harbour Authorithy, 79, 894.

Gripaios, P., \& Gripaios R. (1995). The impact of a port on its local economy: the case of Plymouth. Maritime Policy \& Management, 22(1), 13-24. DOI: 10.1080/03088839500000029.

Grosdidier de Matons, J.C. (1986). Economic and financial appraisal of port projects at the World Bank: a review of policy and practice. Maritime Policy and Management, 13(4), 259-27. DOI: $10.1080 / 03088838600000062$.

Gruen, Gruen \& Associates (1972). An Analysis of the relationship between the port of San Francisco and the Economy of the City and Bay Region. Technical Report Gruen, Gruen \& Associates, San Francisco (USA).

Haddad, E.A., Geoffrey J.D., Hewings, G.J.D., Perobelli, F.S. \& dos Santos. R.A.C. (2010). Regional Effects of Port Infrastructure: A Spatial CGE Application to Brazil. International Regional Science Review, 33 (3), 239-263. DOI: 10.1177/0160017610368690.

Han, L. (2006). A Study On Port Economic Externality Based On System Dynamics. En Globe Thesis C. Recuperado de http://www.globethesis.com/?t=2189330335955446.

Hawkins, J.E. (1991). Port investment appraisals for the 1990's. Maritime Policy and Management, 18(3), 225-232. DOI: 10.1080/03088839100000025.

Hille, S.J. (1975). The economic impact of the Port of Baltimore on Maryland. Division of Transport, Business and Public Policy. Collage of Business and Management. University of Meryland, Meryland (USA).

Hille, S.J. \& Suelflow, J.E. (1969). The economic impact of the Port of Baltimore on Maryland's Economy. Division of Transport, business and public policy. Collage of Business and Management. University of Maryland (USA). Publicado en Proceedings of the $11^{\text {th }}$ Annual Meeting of the Transportation Research Forum (1970), pp: 307-325.

Hong, Z., Merk, O., Nan, Z., Li, J., Mingying, X., Wenqing, X., Xufeng, D. \& Jinggai, W. (2013). Competitiveness of Port-Cities: The Case of Shanghai, China. OECD Regional Development Working Paper 2013/23. París (Francia). DOI: 10.1787/20737009.

Isserman, A.M. \& Merrifield, J. (1982). The use of control groups in evaluating regional economic policy. Regional Science and Urban Economics, 12(1), 45-58. DOI: 10.1016/0166-0462(82)90014$\mathrm{X}$.

Kaufmann, G. (1979). Port Impact Studies: Comparison and evaluation of existing methods. Master Thesis, School of Community and Regional Planning, Universitaet Hamburg. DOI: 10.14288/1.0094568. 
Keynes, J.H. (1936). General Theory of Employment, Interest and Money. Royal Economic Society. Ed. Macmillan Cambridge University Press.

Knapp, J.L., Hammond, J.D. \& Haroz, D.P. (1976). The Impact of Viriginia's Port on the Economy of the Commonwealth. University of Virginia, Taylor Murphy Institute.

Laing, E.T. (1977). The distribution of benefits from port investment. Maritime Policy \& Management, 3(3), 141-154. DOI: 10.1080/03088837700000038.

Leontief, W. (1966). Input-Output Economics. New York, Oxford University Press.

Li, D., \& Wang, X. (2012). System Dynamics Simulation Model for Port Economy Analysis. En Lecture Notes in Electrical Engineering, 185, 475-482. Springer London.

Liu, X., B. Zhao, Li, B., \& Wang, N. (2010). A System Dynamics Model of the Interaction between Port and City Based on the Green Concept. En Proceedings of the CLEM 2010: Logistics For Sustained Economic Development: Infrastructure, Information, Integration y Volume I - Logistics Policy and Strategy (pp: 681-688). Agosto 2010, Chengdu, China. Recuperado de https://ascelibrary.org/doi/pdf/10.1061/41139\%28387\%2995.

MacMullen, J.R. \& Associates, Inc. (1978). The Economic Impact of the Port of Huneme. Port of Huneme, Los Angeles (USA).

MARAD (1978). Economic impact of the U.S. port industry: an input output analysis of waterborne transportation. US Maritime Administration. Office of Economic and Policy Analysis Port Authority of New York \& New Jersey, The Port Department. Nueva York (USA).

MARAD (1982). The Regional Port Impact Model Handbook. US Maritime Administration. Office of Port and Intermodal Development. Washington DC (USA) \& Port Authority of New York \& New Jersey, The Port Department, Nueva York (USA).

MARAD (1995). Economic Impact of the Port Industry on the New York - New Jersey Metropolitan Region. US Maritime Administration. Office of Economic and Policy Analysis Port Authority of New York \& New Jersey, The Port Department, Nueva York (USA).

MARAD (2000). Port Economic Impact Kit (MARAD Port Kit). [CD-ROM]. US Maritime Administration, Department of Transport, Washington DC (USA).

Martin Associattes (2007a). The local and regional impact of the port of Mobile. Alabama State Docks Department. Mobile (Alabama, USA). Recuperado de http://www.asdd.com/aspa_feis/Appendix_C_MobileImpact.pdf.

Martin Associattes (2007b). The local and regional economic impacts of the port of Portland, 2006. Port of Portland (Portland, USA). Recuperado de https://popcdn.azureedge.net/pdfs/Trade_Trans_Studies_Ecnmc_Impact_2006.pdf.

Martin Associattes (2008). The local and regional economic impacts of US Deepwater Port System 2007. American Association of Port Authorities. Recuperado de http://aapa.files.cmsplus.com/PDFs/MartinAssociates.pdf.

Martin Associattes (2012). The local and regional economic impacts of the port of Portland 2011. Port of Portland (Portland, USA). Recuperado de https://popcdn.azureedge.net/pdfs/Trade_Trans_Studies_Ecnmc_Impact_2011.pdf. 
Martin Associattes (2015). The 2014 Economic Impact of the Port of Baltimore. Maryland Port Administration (Maryland, USA). Recuperado de https://popcdn.azureedge.net/pdfs/Trade_Trans_Studies_Ecnmc_Impact_FY15.pdf.

Martin Associattes (2016a). The local and regional economic impacts of the port of Portland. Fiscal year 2015. Port of Portland (Portland, USA). Recuperado de https://popcdn.azureedge.net/pdfs/Trade_Trans_Studies_Ecnmc_Impact_2011.pdf.

Martin Associattes (2016b). The Economic Impact of Port of Corpus Christi, 2015. Maryland Port Administration (Maryland, USA). Recuperado de http://portofcc.com/wpcontent/uploads/CorpusChristi2016_FINAL-1.pdf.

Martin Associattes (2017). Maritime Economic Impact Studies: Seaport Economic Impact Studies. Martin Associattes LLC, Lancaster (Pensilvania, USA). Recuperado de $\mathrm{http} / / /$ martinassoc.co/services/.

Merk, O. (2013a). The Competitiveness of Global Port-Cities: Synthesis Report. OECD Regional Development Working Paper 2013/13. OECD Publishing París (Francia). Recuperado de https://www.oecd.org/cfe/regionaldevelopment/Competitiveness-of-Global-Port-Cities-SynthesisReport.pdf.

Merk, O. (2013b). The Competitiveness of Global Port-Cities: The Case of Antofagasta, Chile. OECD Regional Development Working Paper 2013/15. OECD Publishing. París (Francia). Recuperado de $\mathrm{https}$ ://read.oecd-ilibrary.org/urban-rural-and-regional-development/the-competitiveness-ofglobal-port-cities-the-case-of-antofagasta-chile_5k4067cb267k-en\#page1.

Merk, O., \& Bagis, O. (2013). The Competitiveness of Global Port-Cities: The Case of Mersin-Turkey. OECD Regional Development Working Paper 2013/1. OECD Publishing. París (Francia). Recuperado de https://www.oecd-ilibrary.org/urban-rural-and-regional-development/thecompetitiveness-of-global-port-cities-the-case-of-mersin-turkey_5k4c43014plt-en.

Merk, O., \& Comtois, C. (2012). The Competitiveness of Global Port-Cities: The Case of MarseilleFos, France. OECD Regional Development Working Paper 2011/11. OECD Publishing. París (Francia). Recuperado de https://www.oecd-ilibrary.org/urban-rural-and-regionaldevelopment/competitiveness-of-port-cities-the-case-of-marseille-fos-france_5k8x9b92cnnv-en.

Merk, O., \& Hesse, M. (2012). The Competitiveness of Global Port-Cities: The case of Hamburg, Germany. OECD Regional Development Working Paper 2012/06. OECD Publishing. París (Francia). Recuperado de https://www.oecd-ilibrary.org/urban-rural-and-regional-development/thecompetitiveness-of-global-port-cities-the-case-of-hamburg-germany_5k97g3hm1gvk-en.

Merk, O., \& Li, J. (2013). The Competitiveness of Global Port-Cities: The Case of Hong Kong, China. OECD Regional Development Working Paper 2013/16. OECD Publishing. París (Francia). Recuperado de https://www.oecd-ilibrary.org/urban-rural-and-regional-development/thecompetitiveness-of-global-port-cities-the-case-of-hong-kong-china_5k3wdkjtzp0w-en.

Merk, O., Ducruet, C., Dubarle, P., Haezendonck, E., \& Dooms, M. (2011). Competitiveness of PortCities: The Case of the Seine Axis (Le Havre, Rouen, Paris, Caen) - France. OECD Regional Development Working Paper 2013/23. OECD Publishing. París (Francia). Recuperado de https://www.oecd-ilibrary.org/governance/the-competitiveness-of-global-portcities_5kg58xppgc0n-en;jsessionid=1n2ehf5jg6291.x-oecd-live-01.

Merk, O., Hilmola, O.-P., \& Dubarle, P. (2012). Competitiveness of Port-Cities: the Case of Helsinki, Finland. OECD Regional Development Working Paper 2012/08. OECD Publishing. París (Francia). 
Recuperado de https://www.oecd-ilibrary.org/urban-rural-and-regional-development/thecompetitiveness-of-global-port-cities-the-case-of-helsinki-finland_5k92z70x5v7g-en.

Mulligan. P.F. \& Collins. R.L. (1975). Report of the Impact of the State Economy. North Carolina Department of Transportation and Highway Safety, North Caroline (USA).

Musso, E., Benacchio, M. \& Ferrari, C. (2000). Ports and employment in port cities. International Journal of Maritime Economics, 2(4), 283-311. DOI: 10.1057/ijme.2000.23.

Musso, E., Benacchio, M., Ferrari, C. \& Haralambides, H.E. (2011). On the economic impact of port: local vs national costs and benefits. En Proceedings of the 9th World Conference on Transport Research (WCTR'2011), Seúl (Corea del Sur). Recuperado de https://www.researchgate.net/publication/295402196.

Musso, E., Ferrari, C., \& Benacchio, M. (2006). Port Investment: Profitability, Economic Impact and Financing. Research in Transportation Economics, 16, 171-218. https://doi.org/10.1016/S07398859(06)16008-4.

OECD (2014). The Competitiveness of Global Port-Cities. OECD Publishing, Paris, https://doi.org/10.1787/9789264205277-en

Onyemechi, C., Igboanusi, C.C. \& Ekene-Ezenwa, A. (2014). International trade flow analysis using the gravity model - The Nigirean Conondrum. International Journal of Latest Research in Science and Technology, 3(4), 181-185.

Oregon Department of Transportation (1973). Survey of Oregon ports: Economic Impact Section. Oregon Department of Transportation, Port Division, Oregon (USA).

Opuku, K.A. (1990). The economic impact of the port industry of New York and New Jersey metropolitan region. Port Authority of New York and New Jersey, New York (USA).

Pallis, A.A., Vitsounis, T.K. \& De Langen, P.W. (2009a). Port Economics, Policy and Management: Review of an Emerging Research Field. Transport Reviews, 30(1), 115-161. DOI: 10.1080/01441640902843208.

Pallis, A.A., Vitsounis, T.K., De Langen, P.W., \& Notteboom, T.E. (2009b). A content analysis of published research in port economics, policy and management (1997-2008). En Proceedings of the International Association of Maritime Economists Conference 2009, IAME'09. 24-26 de Junio de 2009, Copenague (Dinamarca).

Pallis, A.A., Vitsounis, T.K., De Langen, P.W., \& Notteboom, T.E. (2011). Port Economics, Policy and Management: Content Classification and Survey. Transport Reviews, 31(4), 445-471. DOI: 10.1080/01441647.2010.530699.

Pender, D.R., \& Wilder, R.P. (1974). Impact of State Ports Authority Upon the economy of South Carolina. Occasional Studies No6. Columbia: Collage of Business Administration. University of South Carolina, South Carolina (USA).

Pinfold, G. (1991). Port of Halifax Economic Impact Study. Port of Halifax, Halifax (Canadá).

Port of Seattle Commission (1971). Seattle Maritime Commerce and its impact on the economy of King County. Planning and Research Development of the Port of Seattle, Seattle (Washington, USA).

Randall, J.E. (1988). Economic development and non-marine initiatives at American Seports. Maritime Policy and Management, 15(3), 225-240. DOI: 10.1080/03088838800000070. 
Rodríguez-Dapena, A. (2005). Una metodología para los estudios de impacto económico de la actividad portuaria. En Proceedings of the XI Congreso de Tráfico Marítimo y Gestión Portuaria (pp. 233262.), 9-14 de Mayo de 2005, Cartagena (Murcia, España).

Roesti, R.M., Coe, R.K., \& Tsagris B, (1964). Economic Impact of the Sacramento -Yolo Port. Mimeo, Sacramento State Collage, Real Estate Research Bureau, Sacramento (California, USA).

Rose, W. (1970). The port of Galveston: Employment and Income Impact. University of Texas, Texas (USA).

Rúa-Ferreño, M., Sastre-Albertí, F., Castro-Hansen, M., Alonso-García, T. \& Tudurí-Egea, M. (2014). El Impacto Económico de los Puertos del Estado en Baleares - 2011 (Versión actualizada con datos del PIB 2013. Ports de Balears (Ministerio de Fomento, Gobierno de España) y Departamento de Economía y Empresas de CAEB (Confederación de Asociaciones Empresariales de Baleares). Recuperado de http://www.portsdebalears.com/sites/default/files/libros/impactoeconmicodelospuertosdelestadoen baleares-151021103747-lva1-app6891.pdf.

Rudy, L.M. (1961). A study of the Economic Impact of Maritime Commerce upon the Port of Seattle District. Port of Seattle, Washington DC (USA).

Ryan, R. \& Adams, C.W. (1973). Corpus Christi. Economic Impact of the Port. Bureau of Business Research, University of Texas, Austin (Texas, USA).

Schenker, E. (1965). Economic of a Port on Urban Community. Transportation Research Forum 1965, New York (USA).

Schenker, E. (1967). The Port of Milwaukee: An economic review. University of Wisconsin, Milwaukee (USA).

Schenker, E. (1972). Impact of the Green Bay on the Economy of the Community. Tech Report 16, University of Wisconsin Sea Grant Program, Milwaukee (USA).

Schenker, E., Tee-Koh, S., Kochan, J., \& Bunamo, M. (1970). An estimation of the quantitative impact on the hinterland's economy. En Proceedings of the $13^{\text {th }}$ Conference on Great Lakes Research (pp: 168-186). Center for Great Lakes Studies, University of Wisconsin, Milwaukee (USA).

Sheng, Q., Ding, T. \& Xu, C. (2013). Research on OD distribution of domestic coastal trade container shipping based on gravity model. En Proceedings of the 6th International Conference on Information Management, Innovation Management and Industrial Engineering (ICIII), 23-24 de noviembre de 2013. Xian (China). DOI: 10.1109/ICIII.2013.6702928.

Stanford Research Institute (1979). The Impact of Sea-Land on the Economy of California. Palo Alto (California, USA).

Stevenson \& Kellog, Ltd (1975). Port of Vancouver: Economic Impact Study. Port of Vancouver. Vancouver, British Columbia (Canadá).

Suykens, F. (1989). The City and its port - an Economic Appraisal. Geoforum, 20(4), 437-445. DOI: 10.1016/0016-7185(89)90027-4.

Temple, Barker \& Sloane, Inc., Recht Hausrath \& Associates \& Regional Science Research Institute (1985). Port Economic Impact Kit. US Maritime Administration, Office of Port and Intermodal Development, Washington DC (USA). 
Tinbergen, J. (1962). Shaping the World Economy: Suggestions for an International Economic Policy. The Twentieth Century Fund, Nueva York (USA).

UK Department of Transport (2018). Wider Economic Benefits Appraisal. Technical Report - Transport Analysis Guidance TAG UNIT A2.1. Transport Appraisal and Strategic Modelling (TASM) Division, Department of Transport. Gobierno del Reino Unido, Londres (UK). Recuperado de https://assets.publishing.service.gov.uk/government/uploads/system/uploads/attachment_data/file/ 712878/tag-unit-a2-1-wider-impacts-overview-document.pdf.

van den Bossche, M., Merk, O., \& Li, J. (2013). The Competitiveness of Global Port-Cities: The Case of Danube Axis (Bratislava, Štúrovo, Komárno), Slovak Republic. OECD Regional Development Working Paper 2013/14. OECD Publishing. París. Recuperado de https://www.oecdilibrary.org/urban-rural-and-regional-development/the-competitiveness-of-global-port-cities-thecase-of-danube-axis-bratislava-sturova-komarno-slovak-republic_5k408j180xr8-en.

Verbeke, A. (1988). The managerial analysis of public infrastructure investment policies. En Van Den Broeck, J. (ed.), Public Choice (pp. 105-144), Kluwer Academic Publishers, Boston (USA). DOI: 10.1007/978-94-015-7784-7_6.

Verbeke, A. \& Debisschop, K. (1996). A note on the use of port economic impact studies for the evaluation of large scale port projects. International Journal of Transport Economics, 23(3), $247-$ 266. https://www.jstor.org/stable/42747551.

Vido, E. (2004). Container Cabotage Policy and it's Impact on Western Canadian Pulse Exports: A Gravity Model Approach. Master Thesis. Department of Agribusiness and Agricultural Economics. University of Manitoba. Winnipeg, Manitoba (Canadá). Recuperado de http://hdl.handle.net/1993/7920.

Villaverde-Castro, J., \& Coto-Millán, P. (1997). Economic Impact Analysis of Santander Port on its hinterland. International Journal of Transport Economics, 24(2), 259-277. Recuperado de https://www.jstor.org/stable/42747294.

Villaverde-Castro, J., \& Maza-Fernández, A. (2015). Competencia y competitividad portuarias: una aplicación a las fachadas marítimas españolas. Revista de Evaluación de Programas y Políticas Públicas. Universidad Nacional de Educación a Distancia (UNED), 4, 59-85. DOI: 10.5944/reppp.4.2015.13348.

Wang, Y. (2010). Analysis of Port Logistics Alliance System based on System Dynamic. En Globe Thesis (C. Recuperado de http://www.globethesis.com/?t=2189360275487279.

Wang, Y., Chou, C.-C. \& Yeo, G.-T. (2013). Application and improvement of a System Dynamics model to forecast the volume of containers. Journal of Applied Science and Engineering, 16(2), 186196. DOI: 10.6180/jase.2013.16.2.10.

Wanhill, S.R.C. (1978). On the cost-benefit analysis of port projects. Maritime Policy \& Management, 5(4), 315-326. DOI: 10.1080/03088837800000022.

Warf, B., \& Cox, J. (1989). The changing economic impacts of the port of New York. Journal Maritime Policy \& Management, 16(1), 3-11. DOI: 10.1080/03088838900000019.

Waters, R.C. (1977). Port Economic Impact Studies: Practice and Assessment. Transportation Journal, 16(3), 4-18. Recuperado de https://www.jstor.org/stable/20712420.

Watson, D.A. (1970). Economic Impact of the Port of Portland, Oregon. Bureau of Business and Economic Research, University of Oregon. Eugene, Oregon (USA). 
Weisbrod, G. \& Weisbrod, B. (1997). Measuring economic impacts of projects and programs. Economic Development Research Group. Boston. Recuperado de http://www.edrgroup.com/pdf/econ-impact-primer.pdf.

Whitaker-Mohn, A. (1974). Economic Impact of the Port of Sacramento: Its Sources and Results. California State University. California (USA).

Williams-Kuebelbeck \& Associates, Inc. (1976). Economic Impact of Waterborne Commerce through the Ports of Los Angeles and Long Beach. Williams-Kuebelbeck \& Associates, Inc., Marina del Rey (California, USA).

Williams-Kuebelbeck \& Associattes, Inc. (1978). Washington Public Ports Economic Study. Marina del Rey (California, USA).

Xiaodong, L., Zhao, B., Li, B., \& Wang, N. (2010). A System Dynamics Model of the Interaction between Port and City Based on the Green Concept. En Proceedings of the ICLEM 2010 - Logistics For Sustained Economic Development: Infrastructure, Information, Integration. Vol I - Logistics Policy and Strategy, pp. 681-688. Agosto 2010, Chengdu (China).

Xing S. (2012). Research on the regional economic contribution of Port of Tianjin. En Globe Thesis C. Recuperado de http://www.globethesis.com/?t=2189330335455698.

Yang Y.B., \& Liu, B. (2011). A new method of Container Port Hinterland Division. Advance Material Research, 403-408, 3661-3665. DOI: 10.4028/www.scientific.net/AMR.403-408.3661.

Yang, J.-Q. (2002). Gravity model for partitioning port hinterlands based on fuzzy comprehensive evaluation. Journal of Traffic and Transportation Engineering, 2(2), 123-126.

Yang, X.J. (2012). Port Logistics Strategic Alliance Research. En Globe Thesis C. Recuperado de http://www.globethesis.com/?t=2189330335455484.

Yang, Y. (2007). Study on the impact of port for regional economy based on System Dynamics Model. En Globe Thesis (C. Recuperado de http://www.globethesis.com/?t=2179360182972089.

Yang, Y.B., Liu, B., \& Zhang, B. (2012). The Study on Hinterland Relevant to Container Port. Applied Mechanics and Materials, 170-173, 2284-2287. DOI: 10.4028/www.scientific.net/AMM.170173.2284 .

Yochum, G.R., \& Agarwal, V.B. (1987). Economic impact of a port on a regional economy. Growth and Change, 18(3), 74-87. DOI: 10.1111/j.1468-2257.1987.tb00082.x.

Yochum, G.R., \& Agarwal, V.B. (1988). Static and changing port economic impacts. Maritime Policy and Management, 15(2), 157-171. DOI: 10.1080/03088838800000026.

Yu, B., Zhang. C., Kong, L., Bao, H.-L., Wang, W.-S., Ke, S., \& Ning, G. (2014). System dynamics modeling for the land transportation system in a port city. Simulation, 90(6), 706-716. DOI: $10.1177 / 0037549714533619$.

Zhang, M. (2010). Study on the Relationship between the Development of Port Industry and City Based on Green Conception. En Globe Thesis (C) Recuperado de http://www.globethesis.com/?t=2189360275453580.

Zhao, B. (2009). Study on Green Contribution of Port to City Based on System Dynamics. En Economic Papers. Recuperado de http://www.economics-papers.com/study-on-green-contribution-of-port-tocity-based-on-system-dynamics.html. 